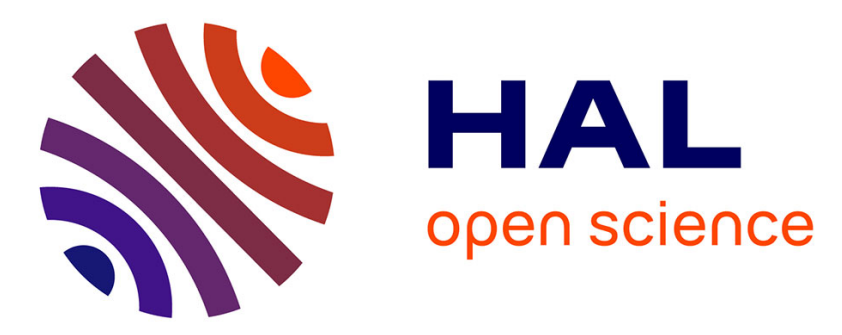

\title{
Aspects et problèmes morphologiques du massif de Devon-Cornwall comparés à ceux d'Armorique
}

\author{
André Guilcher
}

\section{To cite this version:}

André Guilcher. Aspects et problèmes morphologiques du massif de Devon-Cornwall comparés à ceux d'Armorique. Revue de Géographie Alpine / Journal of Alpine Research, 1949, XXXVIII (IV) (4), pp.689-717. 10.3406/rga.1949.4048 . insu-02276835

\section{HAL Id: insu-02276835 \\ https://hal-insu.archives-ouvertes.fr/insu-02276835}

Submitted on 8 Feb 2021

HAL is a multi-disciplinary open access archive for the deposit and dissemination of scientific research documents, whether they are published or not. The documents may come from teaching and research institutions in France or abroad, or from public or private research centers.
L'archive ouverte pluridisciplinaire HAL, est destinée au dépôt et à la diffusion de documents scientifiques de niveau recherche, publiés ou non, émanant des établissements d'enseignement et de recherche français ou étrangers, des laboratoires publics ou privés. 


\section{Aspects et problèmes morphologiques du massif de Devon- Cornwall comparés à ceux d'Armorique} André Guilcher

\section{Citer ce document / Cite this document :}

Guilcher André. Aspects et problèmes morphologiques du massif de Devon-Cornwall comparés à ceux d'Armorique . In: Revue de géographie alpine, tome $37, n^{\circ} 4,1949$. pp. 689-717;

doi : https://doi.org/10.3406/rga.1949.4048

https://www.persee.fr/doc/rga_0035-1121_1949_num_37_4_4048

Fichier pdf généré le 20/04/2018 


\title{
ASPECTS ET PROBLÈMES MORPHOLOGIQUES DU MASSIF DE DEVON-CORNWALL COMPARÉS A CEUX D'ARMORIQUE
}

\author{
par André GUILCHER
}

En septembre 1948 j'ai pu, grâce au C.N.R.S., étudier pendant trois semaines le relief du Massif de Cornwall-Ievon ${ }^{1}$. Je voulais surtout le comparer au Massif Armoricain et spécialement à ia Bretagne. Le massif du SW britannique est celui du monde qui ressemble le plus à l'Armorique, même sans faire état de son appartenance celtique; leurs différences n'en sont que plus intéressantes à préciser. Cornouaille, Domnonée d'Outre-Manche et leur bordure mériteraient de fixer l'attention d'un jeune morphologiste en quête d'un sujet de thèse. Car on ne peut prétendre

1 Je remercie les personnes qui mont aide à des titres divers : en (irandeBretagne, les Professeurs L. Dudley Stamp (Universite de Londres), Davies et Kidson (Cniv. d'Excter); en France, M. le Doyen Cholley (Sorbonne), M. Teissier (Directeur du CNRS), M. Cailleux (Professeur à l'Ecole des Hautes Etudes); je n'ai garde d'oublicr les Directeurs de la Revue de Géographie Alpine, grâce à lobligeance desquels eet article peut voir le jour.

Orientation bibliographique. Les deux travaux de base suivants donnent de nombreuses refirences : H. Dewey, South West England. IBitish Regional Geology, Londres, $2^{e}$ ed., 1948, in-8 ${ }^{\circ}, 72$ p.; - J. A. Steers, The Coastline of Fngland and Wales. Cambridge Cniv. Press, 2\% ed., 1948, in-8\%, 64t p. - Ie chapitre des Iles Britanniques de Demangeon sur la région est un des meilleurs de l'ouvrage. - Voir aussi les références aux pages qui suivent.

Abrébiations employees : .J(iS : Quarterly Journal of the licological Society; PGA : Procedings of the Geologists' Association; - EGS : Explanations of the sheets of the Geological Survey of lingland and wales.

Cartes topographiques : 1 pouce/4 miles (253440"), Fngland and Wales, nos 10 et 11 . - 1 pouce/ 1 mile $\left(63360^{\circ}\right), n^{\prime \prime} 163$ à 165,174 à 177,185 à 190 . Nombreux 25000\%. - Cartes géologiques: 1 pouce/1 mile, liste dans Iewey (stocks et matrices détruits par bombardements, feuille d'Exeter seule réditéc en sept. 1948). - 1 pouce/10 miles $\left(633600^{*}\right)$ Great Britain, n" 2, 1948. 
apporter des solutions définitives après un court voyage comme le mien; les figures 1,5 a et 5 b sont des essais, et je dois naturellement à la littérature antérieure le meilleur de ce que je puis dire ici. On peut seulement poser des problèmes, donner son avis sur d'autres déjà discutés : venant d'un pays où les morphologistes sont formés sous d'autres influences, une opinion nouvelle peut avoir son intérêt même après une visite rapide. Les développements seront réduits au mininum; on a préféré y suppléer par la figuration, plus expressive qu'un long texte.

\section{I. - La bordure orientale du massif ancien.}

On cherche souvent dans les bordures secondaires des massifs anciens la clé des problèmes de leur relief intérieur, parce que les dépôts de ces bordures peuvent fossiliser des surfaces qui se poursuivent, exhumées, sur les terrains primaires et cristallins. Aussi commencera-t-on par l'E, où Dévonien et Carbonifère passent sous le New Red Sandstone (nouveau grès rouge) du Permo-Trias.

Cette bordure offre deux sections assez distinctes de part et d'autre du méridien de Wellington (fig. 1). Au N, la complexité est extrême. Le massif y est formé de Dévonien à facies variés. Les Foreland Grits èt les Hangman Grits sont des roches d'ordinaire très dures, où dominent nettement des grès cohérents à grain fin ressemblant assez au grès armoricain de Bretagne et de Normandie. Les Lynton Beds et Ilfracombe Beds sont bien plus schisteux. Ce matériel a été affecté de failles très nombreuses, généralement NW-SE ou WNW-ESE, qui ont donné un régime de blocs basculés subparallèles non sans analogie avec le Màconnais. Le plus grand bloc est celui des Quantock Hills, îlot de roches primaires dont les fig. 1,2 et $3 \mathrm{~A}$ montrent la disposition et les variations du NW au SE et qui, de ses 383 mètres, offre un beau point de vue sur le Pays de Galles. Plus au NW, Grabbist Hill et North Hill, que je n'ai pas visitées mais que Thomas 2 a étudiées, sont aussi basculées au NE et présentent, au SW et au $\mathrm{S}$, des escarpements de faille exhumés, en grande partie au moins, du New Red Sandstone. Mais il ne faudrait pas croire qu'on peut suivre très loin, par prolongation de profils, la surface sortant de dessous

2A. N. Thomas, The Triassic Rocks of NW Somerset. PGA, LI, 1940, p. 1-43. - Sur les Quantocks: W. A. E. Ussher and H. B. Woodward, The Geology of the Quantock Hills... EGS, 1908, 109 p. 


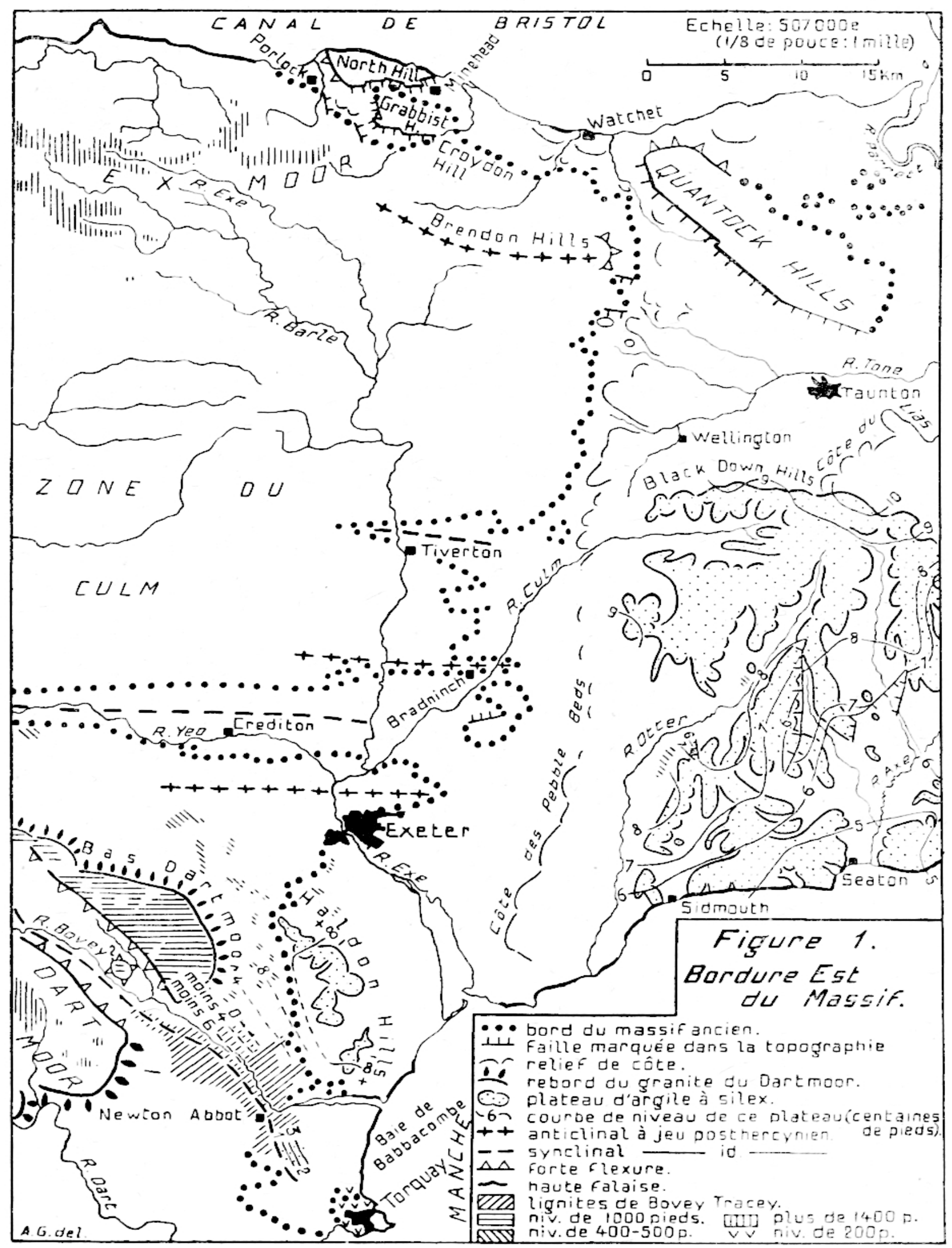


le New Red sur le revers des blocs. Ies failles ont fonctionné arant, pendant et après le dépòt du New Red; et la surface de base de celui-ci s'accidente fortement, d'après Thomas, de vallées et de dèpressions de sédimentation permo-triasique. Il n'y a donc pas grand'chose à tirer de cette région pour la datation des formes du massif. Notons seulement que le Permo-Trias s'est étendu loin vers l'W, par le bord du massif sinon par l'intérieur, puisqu'il en existe un témoin sur la cóle cntre Barnstaple et la lointe de Hartland (NW Devon).

La bordure au $S$ de Wellington nous retiendra un peu plus. Si les Quantock Hills sont une Forêt de Perseigne, les Black Down Hills et les plateaux disséqués les continuant jusqu'à la Manche sont un Perche (fig. 1 et 3 I) : . Le soubassement de ces plateaux est formé, tantôt de grès crétacés du Greensand, tantôt de craie, tous deux fortement discordants sur le 'Trias. Mais ils sont plaqués de formations qui en font avec évidence une surface d'érosion : avant tout l'argile à silex qui, à Salcombe Hill près de Sidmouth, est consolidée en un conglomérat à pâte très dure, véritable grès siliceux fin quand les silex sont peu abondants : c'est une formation identique aux conglomérats de silex de la Butte de l'Orme au Loup près de Sancerre, ou encore du plateau d'entre Laigle et Verneuil sur le revers oriental du Perche. De plus, les Black Downs portent souvent des cailloutis roulés de silex, quartz, grès bleus fins, qu'on retrouve par endroits entre là et la Manche. Enfin, à Castle Neroche (SSW de Taunton), le plateau recèle un gros bloc résiduel de grès, et plus au $N$ il $y$ en a d'autres, descendus sur place au cours du déblaiement du Crétacé : ces sarsens ou greywethers à peine entaillables au marteau me sont apparus comme les correspondants des Grès à Sabals, qui parsèment une vaste surface d'érosion très aplanie datant du début du Tertiaire et sont visibles en de très nombreux endroits du Massif Armoricain et de sa bordure mancelle et angevine ${ }^{4}$. Il est certain que le plateau est une surface éocène ou au moins éogène originellement très plane, qui dans son état actuel (fig. 1) descend vers la Manche et est disloquée par endroits par des failles dont le rejet peut approcher de

3 Ussher and Woodward, The Geology... between Wellington and Chard. EGS, 1906,68 p. - Id., ibid., The Geology... near Sidmouth and Iyme Regis. EGS, 1906,96 p.

4 Cf. notamment R. Musset, Ie relief du Haut Maine. Ann. de Géogr., XXXVIII, 1929, p. 305-329. - A. Guilcher, I.e relief de la Bretagne méridionale. 'Thèse, La Roche-sur-Yon, 1948, 682 p. (conditions climatiques de formation, p. 534-539; voir aussi passim, et bibliogr.). 


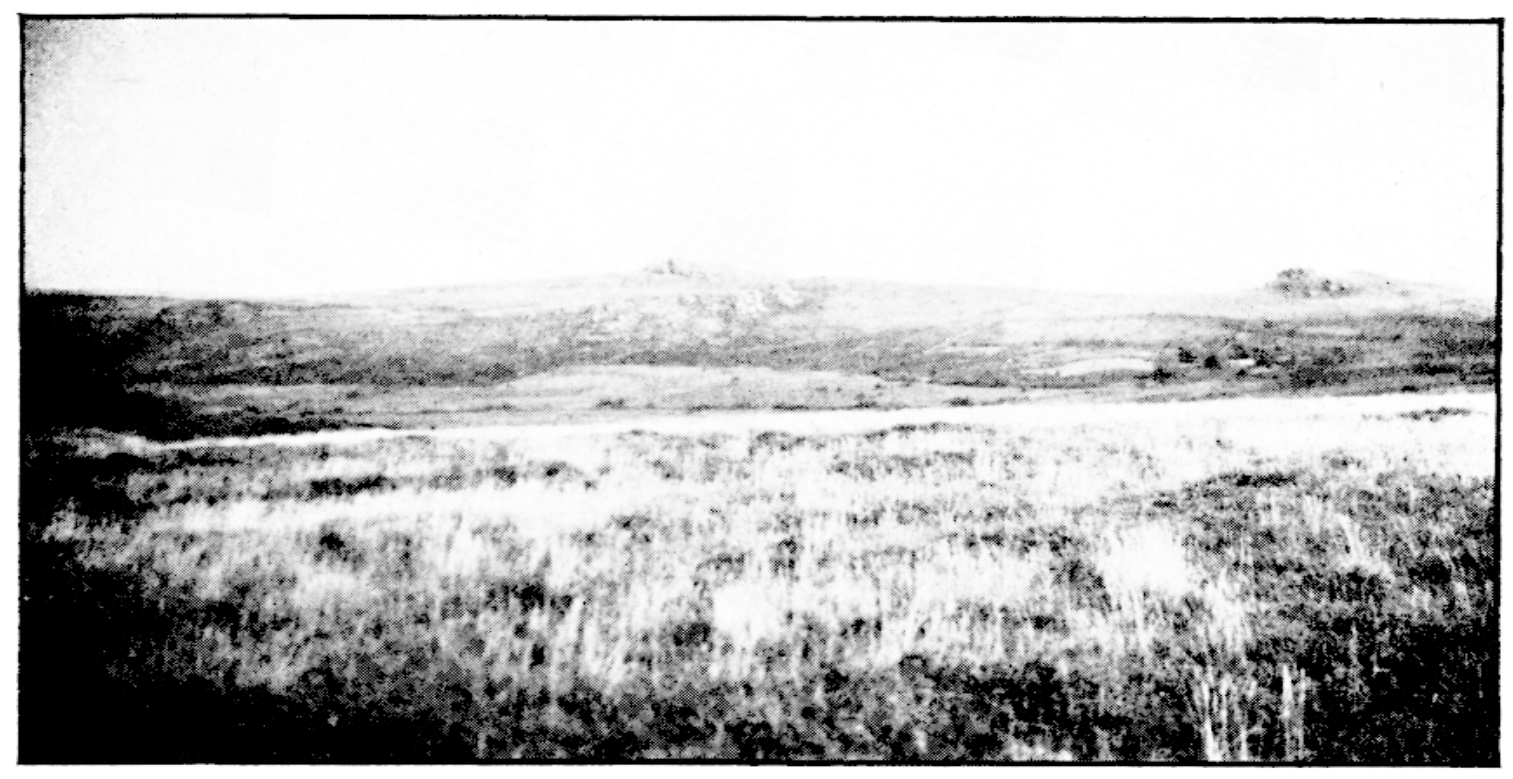

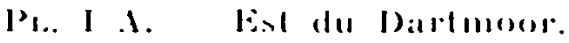

Tors de granite (Holwell Tor det Haytor Rocks). Hande a brugeres ed at forugeres rpas d'ajoncs).

\section{P.. I B. R. Bmwn Willy Tor, point culminanll

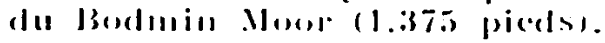

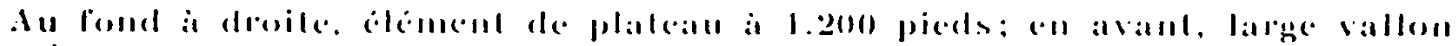

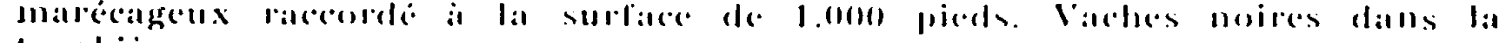
tourhicier. 


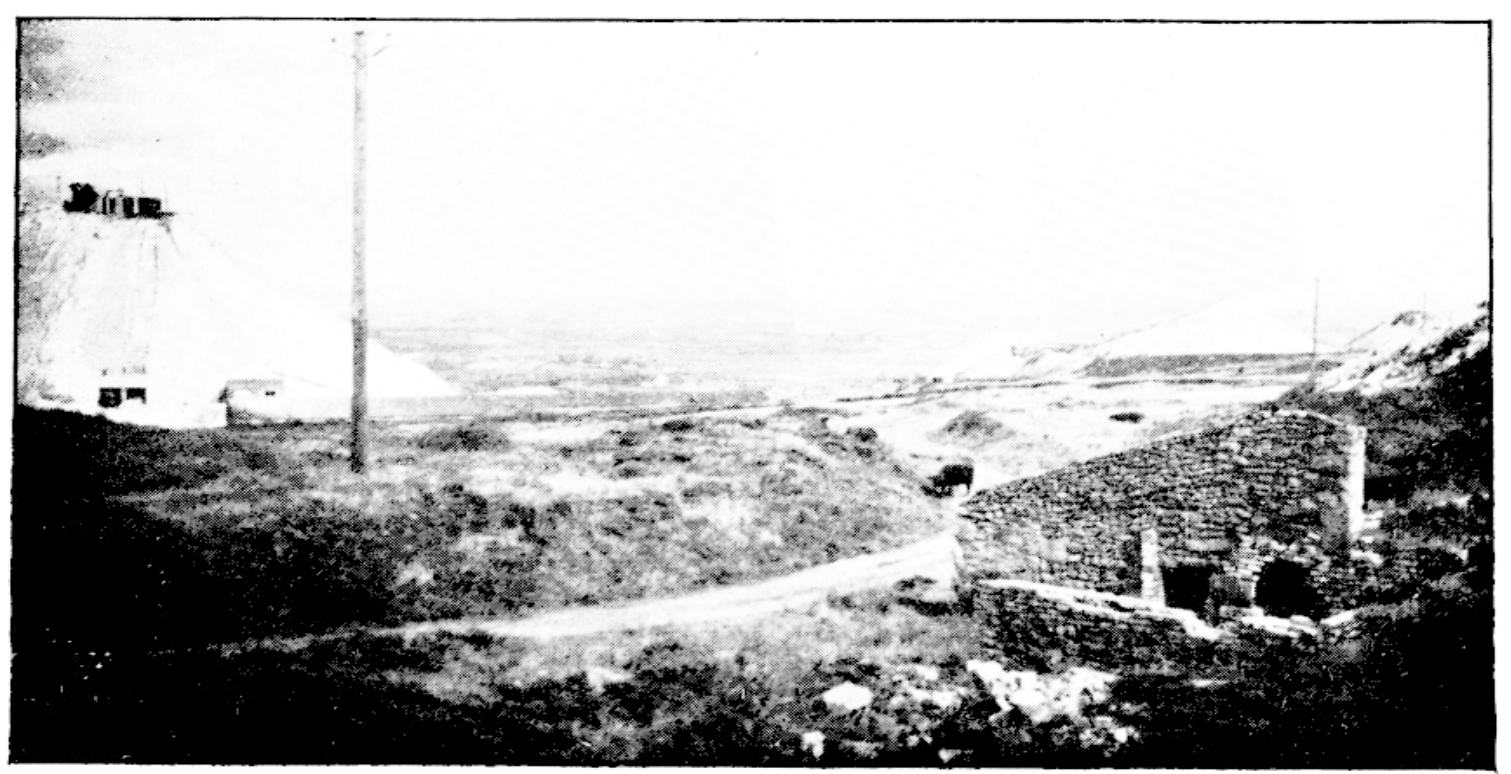

Pe. Il A. -- Retombie Nli des Hensbarmow Downs (massif de St Austell?.

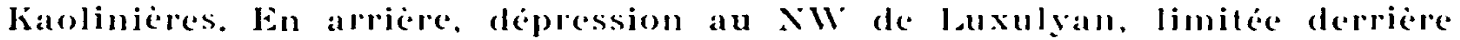
le crassier de droite par des collines granitifues. Ie Bodmin Mone se devine au fond a gatuche.

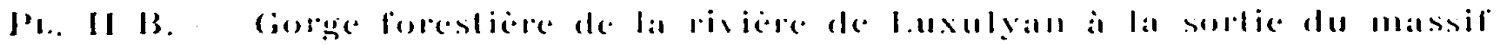

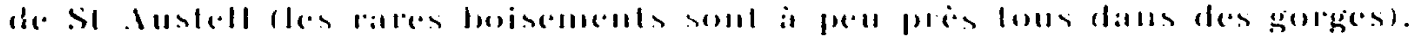




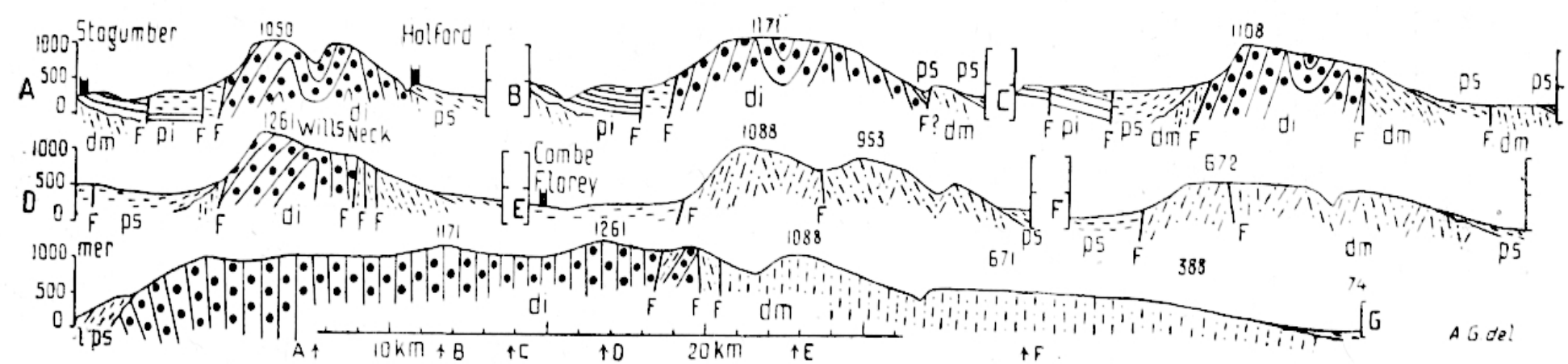

Fig. 2. - Quantock Hills. Coupes transversales SW-NE (A à F) et coupe longitudinale $\mathrm{NW}$-SE (G). Passages des petites coupes sur la grande indiquées au bas de cette dernière. Hauteurs en pieds, longueurs en kilomètres. - di : Dévonien inférieur (Hangman (irits). - $\mathrm{dm}$ : Dévonien moven (schistes et grès). - pi : Permo-Trias inféricur (n'existe qu'au $\mathrm{SW}^{\prime}$ ). - ps : Permo-Trias supérieur. - l : lias. - F : faille. - Plissement conventionnel pour le Dévonien. 
$100 \mathrm{~m}$." Le lecteur familier avec les paysages et problèmes de l'extrême Ouest du Bassin de Paris les retrouvera ici : dépôts superficiels, dislocations de la vieille surface, plateaux disséqués en longues lanières, tout $y$ est, sauf l'harmonie du bombement percheron ${ }^{6}$.

Aux approches du massif, le plateau a été détruit et, au-delà du bord concave du Greensand, c'est une dépression périphérique développée dans les marnes, sables et conglomérats du PermoTrias. Jans cette plaine au sol de couleur lie de vin, les Pebble Beds (galets) du Bunter (grès bigarré, Buntsandstein) donnent une côte fuyante et molle, qui est loin d'atteindre le commandement du plateau d'arģile à silex parce que les galets sont à peine liés entre eux, mais qui, d'un point élevé comme le site de l'austère colonne du Duc de Wellington sur les Black Downs, apparait avec une continuité presque complète ${ }^{7}$. Mais au SSW d'Exeter, les. Haldon Hills à la silhouette tabulaire portent jusqu'à plus de 800 pieds sur leurs Greensands un très large témoin d'argile à silex à $20 \mathrm{~km}$ à l'W du plateau principal.

La surface du début du Tertiaire arrive donc ici très près du massif ancien, comme aussi la surface de contact du Crétacé et du Permo-Trias qui est, on l'a vu, un plan d'érosion. Pénètrent-elles dans le Primaire? Déception là encore. De Wellington à Exeter, le bord du massif s'accidente d'anticlinaux et synclinaux W-E qui affectent le Culm (schistes carbonifères) et sa couverture de PermoTrias (fig. 1). La surface prépermienne paraît bien avoir èté ici une pénéplaine ${ }^{8}$; mais avec une tectonique si compliquée et un matériel primaire si aisément disséqué, il serait sans doute osé de prolonger sur quelque étendue surfaces prépernłienne, précrétacée ou éocène en dehors de leurs terrains de recouvrement. En un endroit seulement on peut préciser un peu. Vers Newton Abbot et Bovey Tracey, un très profond synclinal contient des argiles à lignites oligocènes jusqu'à plus de $200 \mathrm{~m}$. sous le niveau de la mer; dans le $\mathrm{S}$ de cet accident, à Aller, j'ai visité des argiles à silex et à galets de quartz et roches primaires, qui ont au moins $30 \mathrm{~m}$.

5 Il me parait difficile de distinguer avec Green (PGA, LII, 1941, p. 36-52) une série de niveaux dérosion récents dans ce plateau. Les faibles gradins qu'on observe par endroits n'entament guère l'argile à silex; les déformations tertiaires en rendent suffisamment compte. C'est plutôt en contre-bas du platcau qu'il $y$ aurait des formes cycliques emboîtées. Cf. infra.

6 Cf. R. Musset, Le relief du Perche. Ann. de Géogr., XXIX, 1920, p. 99-126.

7 A $N$ de Wellington, les conglomérats du New Red inférieur donnent aussi des ébauches de côtes (fig. 1).

8 A. Guilche:, Remarques sur la surface posthercynienne. Ann. de Géogr., 1949 (sous presse). 


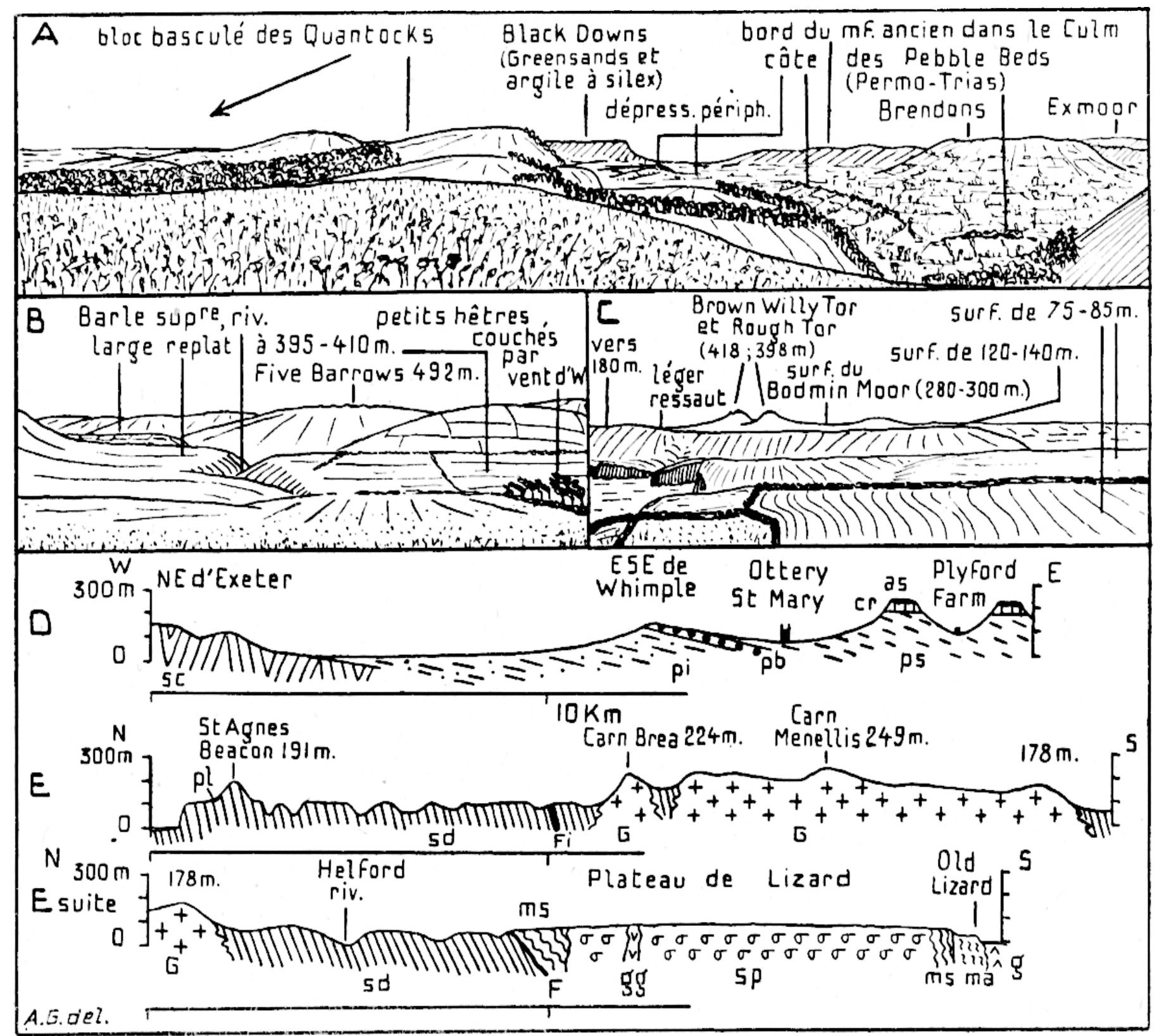

Fig. 3. - Bord du massif ancien et niveaux étagés (A,B,C, d’après nature).

A. De Ia cote 1171 (pieds) all-dessus de Crowcombe dans les Quantock IIills rers l'W et le $\mathrm{S}$. - B. De Wood Barrow

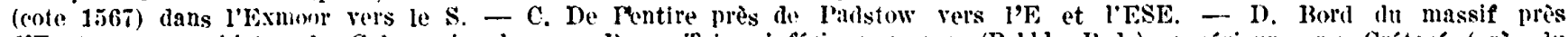

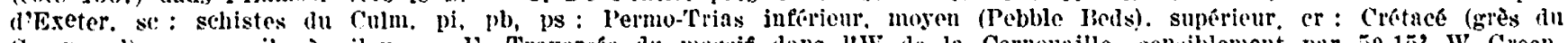

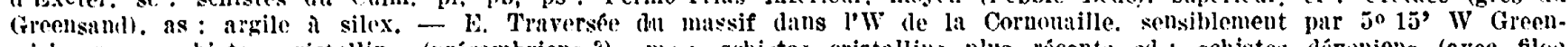
wich. ma: schistes cristallins (précambriens?). ms: schistes cristallins plus récents sol : schistes dívoniens (avec filon

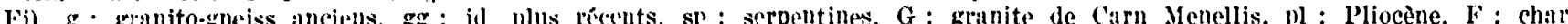
riage de Iizalld. 
d'épaisseur et témoignent du ploiement de la surface cocène par le synclinal. Celui-ci affecte très nettement la partie NF du sranite du Dartmoor en y déterminant le large sillon de Morelonhampstead (fig. 1, 4, 5 a), (fui isole au NE le plateau qu'on peut appeler Bas Dartmoor". Mais, si l'on suit bien jusqu'au bord du l)artmoor la surface éocène déformée et fossilisée par les liggnites, comment la retrouver plus loin, puisqu'une tectonique tertiaire difficile à déceler se superpose certainement, là encore, à la tectonique primaire? Il faudrait découvrir des lambeaux de couverture ćogène sur le massif; jusqu'ici, personne n'en a décrits 1". Les mouvements très amples et violents enregistrés par ce massif, ainsi que son absence apparente de couverture, le rendent donc bien plus délicat à étudier que le Massif Armoricain, fortement gondolé et faillé au 'Tertiaire comme l'ont montré divers travaux récents ${ }^{11}$, mais bien moins tout de même. Nous sommes plus loin des Alpes, sans doute, mais peut-être sous l'influence plus directe des centres perturbateurs atlantiques dont témoignent les montagnes galloises, écossaises et scandinaves. Car tous ces massifs du NW européen, qui descendent doucement vers le bassin franco-britannique et la plaine russe mais sont tranchés par la mer à l'W de façon plus brutale, ne doivent sans doute guère aux convulsions mésogéennes, mais beaucoup à celles que nous cache l'Océan. De la pointe de la Bretagne au Cap Nord, c'est vers l'Ouest qu'il faut regarder.

Il reste qu'on peut sans doute retracer des étapes de l'ividement du New Red, et de celui du Culm entre les Haldons et le Dartmoor. Il semble $y$ avoir des restes d'un niveau de $400-500$ pieds s'élevant vers le $N$. I.es indications de la fig. 1 ne sont appuyces que sur des observations rapides. Plus bas existent encore d'autres niveaux d'après les auteurs anglais ${ }^{12}$. Dans cet ordre d'idées, une etude détaillée de toute la bordure arriverait probablement à un résultat d'ensemble assez net, car ces niveaux ne paraissent guère déformés.

'Sur ce synclinal et les Haldons, ef. Jukes-Browne, .MGS, LX, 1904, p. 319334 , et IXIII, 1907, p. 106-123.

1" Quant au Permo-Tiais, il se retrouve par lambeaux jusquà Plymouth. Il y cu a certainement des gisements immergés devant Lizard point (Worth, JGS, XIII, 1886 , p. 313-315; Dangeard, Observations de géologie... relatives a la Manche. Ann. Inst. Ocértnogr., VI, 1, 1928, 295 p.). Mais tout ce qui est visible est toujours trop failé pour servir à dater une large surface.

11 Guilcher, these, passim, et bibliographic (notamment à Meynier).

1:.J. I. N. Green, The Terraces of Southernmost England. JGS, XCII, 1936, p. Lvil-ixxxili (p. Lxxvin et Lxxxil). - Au $N$ : Thomas, op. cit., p. 5-7. 


\section{II. - Problemes des points rolminants du massif.}

Avant même d'entrer dans le massif ancien, on voit des Haldons ou des Quantocks les hauts et lourds bossellements du Dartmoor et de l'Fxmoor, qui annoncent les autres déserts de bruyères, de fougères et de tourbe du Bodmin Moor, de St Austell, Carn Menellis et Land's End. Voici le grand contraste avec le Massif Armoricain. En-deçà de la Manche, le cristallin ne forme jamais, au moins dans le $S$ et l'W, les zones culminantes, sauf exception due à la tectonique (Montagne de Locronan près de Douarnenez). Il est nivelé par la surface éocène. Ici, seul l'Exmoor se dresse dans le Jévonien; par ailleurs, rien que du granite aux sommets. Les petits pointements cristallins donnent, comme les grands, à peu près toujours des hauteurs : tels Kit Hill au NNW de Plymouth; Castle an Dinas, Belowda Beacon et Roche près du massif de St Austell; le Mont Saint Michel de Penzance, etc. I'île de Iundy et les Scillies sont aussi en granite. Ises rebords topographiques ont seulement parfois reculé vers l'intérieur des massifs.

Il $y$ a là deux problèmes distincts : pourquoi le Sédimentaire ne donne-t-il pas ici tous les points culminants? Pourquoi le Granite n'est-il pas nivelé ?

A la première question, on peut répondre que, sauf justement dans l'Exmoor, le matériel paléozoïque est toujours à prédominance schisteuse. Ia seule roche vrament comparable au grès armoricain (Ordovicien) et aux dalles ardoisières du Gédinnien (Dévonien inférieur) qui constituent l'ossature des principaux reliefs de Normandie, celle des Monts d'Arrée et de la Montagne Noire de Bretagne, ce sont les Staddon Grits, grès et quartzites du Dévonien inférieur qui forment une bande W-E morcelée mais traversant tout le massif du $N$ de Newquay au $N$ de Dartmouth. Or cette bande, renforcée parfois des bancs gréseux des Dartmouth Slates (ardoises de Dartmouth), donne souvent une ligne de hateurs: St Breock Downs dans l'W (fig. 7 et 5 b); crêtes encadrant la baie de Plymouth et l'estuaire de la I)art (fig. 5 a et 9 F). Mais ces hauteurs restent très inférieures aux culminations granitiques, et la rèponse est insuffisante. Peut-être cette bande était-elle trop étroite pour conserver une grande altitude ${ }^{1: 3}$.

1:3 Par ailleurs, les adaptations structurales ne sont pas totalement absentes: au $N$ des schistes métamorphiques de lizard et de Start sesquissent (fort mal), dans des sehistes plus tendres, des dépressions où s"épanouissent la Helford River et l'estuaire ramifie de Kingsbridge (ce dernier et celui de plymouth sont du type du Trieux en Bretagne, avec entréc retrécic dans des roches (lures); au SE et à l'W (lu Jartmoor, des diabases et roches volcaniques domnent des chauches de crètes, ete. (fig. 5 a). Mais c'est peu de chose a côli des reliefs appalachicns armoricains. 
Quant à l'Exmoor, il n'est sans doute pas sans rapport avec la présence des Hangman Grits ${ }^{14}$. Les hauteurs de plus de 1400 pieds (425 m.) débordent largement, il est vrai, sur les schistes d'Ilfracombe et de Lynton, et ne s'étendent pas à toute la bande de Hangman Grits (of. infra, $3^{\mathbf{e}}$ partie); mais l'adaptation à la rćsistance du soubassement n'est pas nulle. Les rivières (fig. 1) tendent à s'aligner, entre des mamelons encore plus lourds que ceux des massifs cristallins, selon la direction WNW-ESE des couches. En outre, sur la haute Barle ainsi orientée, j’ai observé (fig. 3 B) de très nets replats, témoins d'un stade de creusement. Cette haute région devrait donc en partie son altitude à la présence de la plus grande masse sédimentaire dure de tout le massif, mais l'évolution de type appalachien est encore très vague. Tout cela ne nous dit pas pourquoi l'Exmoor est plus bas que le Dartmoor cristallin. Un examen plus complet conduira peut-être à arguer de mouvements tertiaires.

Les altitudes atteintes dans le granite pourraient en effet s'expliquer ainsi. En raisonnant par analogie avec le $S W$ du Massif Armoricain, où les reliefs prééocènes n'occupent qu'une place infime ${ }^{15}$, il n'est pas absurde de supposer que la pénéplaine éocène, attestée sur la bordure $\mathrm{E}$, avait nivelé. granites et roches sédimentaires dans leur ensemble, sauf les grès du $\mathbf{N}$ du Devon (Exmoor), et peut-être sans excepter les Staddon Grits, trop peu larges pour résister. Cette pénéplaine fưt, on l'a vu, extrêmement bouleversée, et sans doute portée bien plus haut que dans le Massif Armoricain. Cela détermina une reprise d'érosion très vive, qui détruisit les témoins de cette pénéplaine dans toutes les régions schisteuses, de beaucoup prédominantes; les massifs cristallins, ou bien conserveraient des restes de cette surface comme en Bretagne, ou bien en seraient des éléments plus ou moins surbaissés ou repris dans des surfaces postérieures, mais plus élevées que celles nivelant les régions avoisinantes.

Un contrôle de cette hypothèse de travail consisterait à chercher des débris de couverture éocène sur le Dartmoor ou le granite de St Austell, les deux massifs où, à notre avis, on aurait le plus de chanees d'en trouver. Mais il faut probablement écarter les kaolins pourtant si abondants, car ils sont tous, d'après les auteurs, d'origine profonde, au contraire de ce qui se passe en Bretagne 15 bis.

14 Sur ces roches, cf. 1 re partie, début.

15 Guilcher, thèse, passim.

15 bis 'Toutefois, j'ai vu des argiles kaoliniques associées à la formation à silex dans la carrière d'Aller près de Newton Abbot. Elles peuvent remanier des kaolins profonds du Dartmoor. 
Ces massifs ne sont d'ailleurs pas tous semblables; mais deux. seulement peuvent être examinés en dehors du problème des aplanissements étagés.

Le massif de St Austell (fig. $5 \mathrm{~b}$ et 6) est une grosse intumescence granitique (312 m. au Hensbarrow Beacon) à qui l'intensité

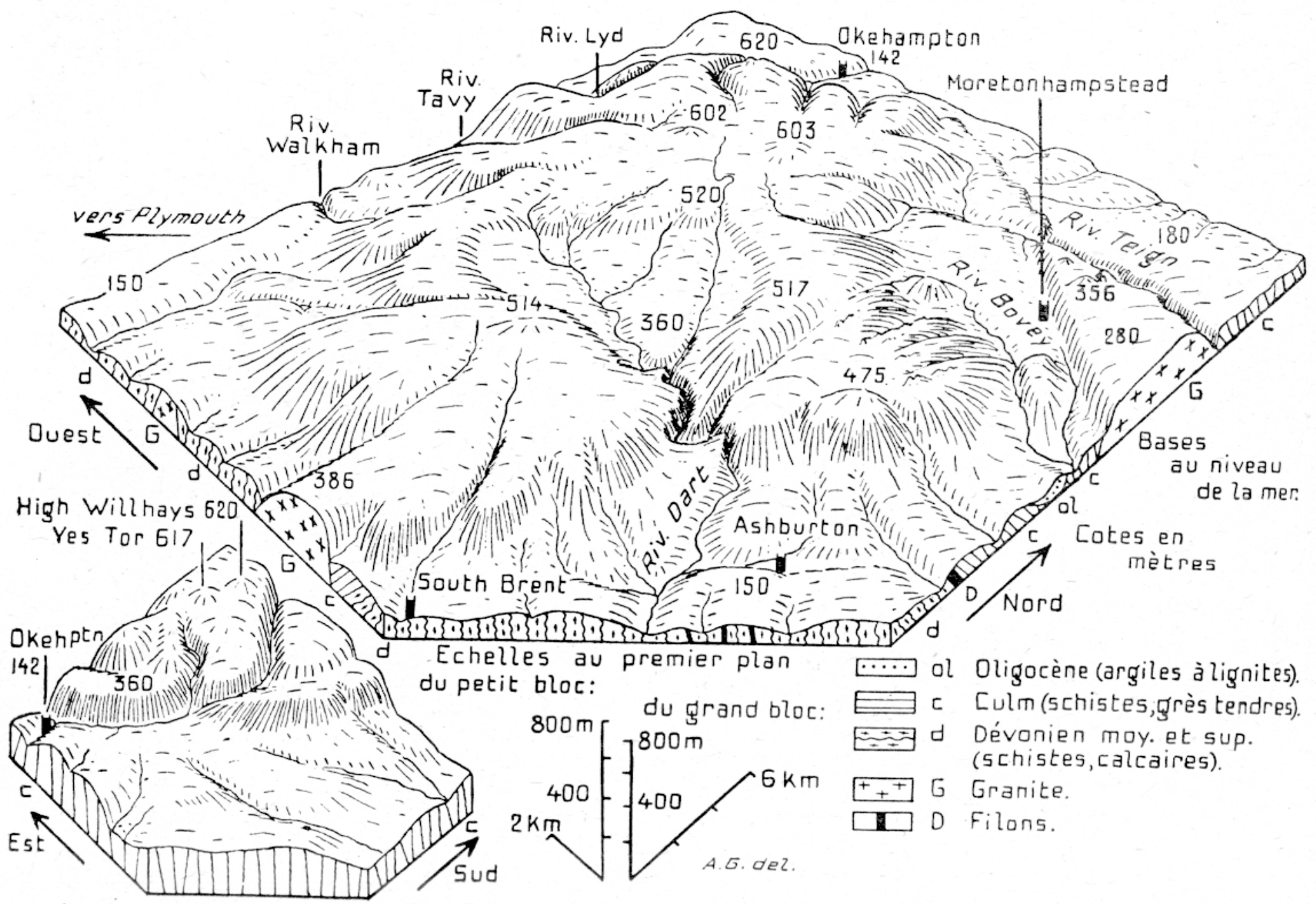

Fig. 4. - Blocs diagrammes du Dartmoor (le petit correspond à l'arrière du grand; leurs perspectives sont en sens inverses). Front montagneux du $\mathrm{N}$, cuvette centrale, gorge de la Dart, synclinal de Bovey Tracey au NE.

des processus pneumatolytiques dont elle fut le siège vaut d'être le premier centre kaolinier du monde : une centaine de crassiers d'un blanc éclatant, plus ou moins ravinés selon leur ancienneté, y composent un étrange paysage d'allure volcanique. De l'W, de l'E et $\mathrm{du} S$, les pentes s'élèvent assez régulièrement vers les bosses du sommet. Mais au NE s'ouvrent dans le granite, non kaolinisé 


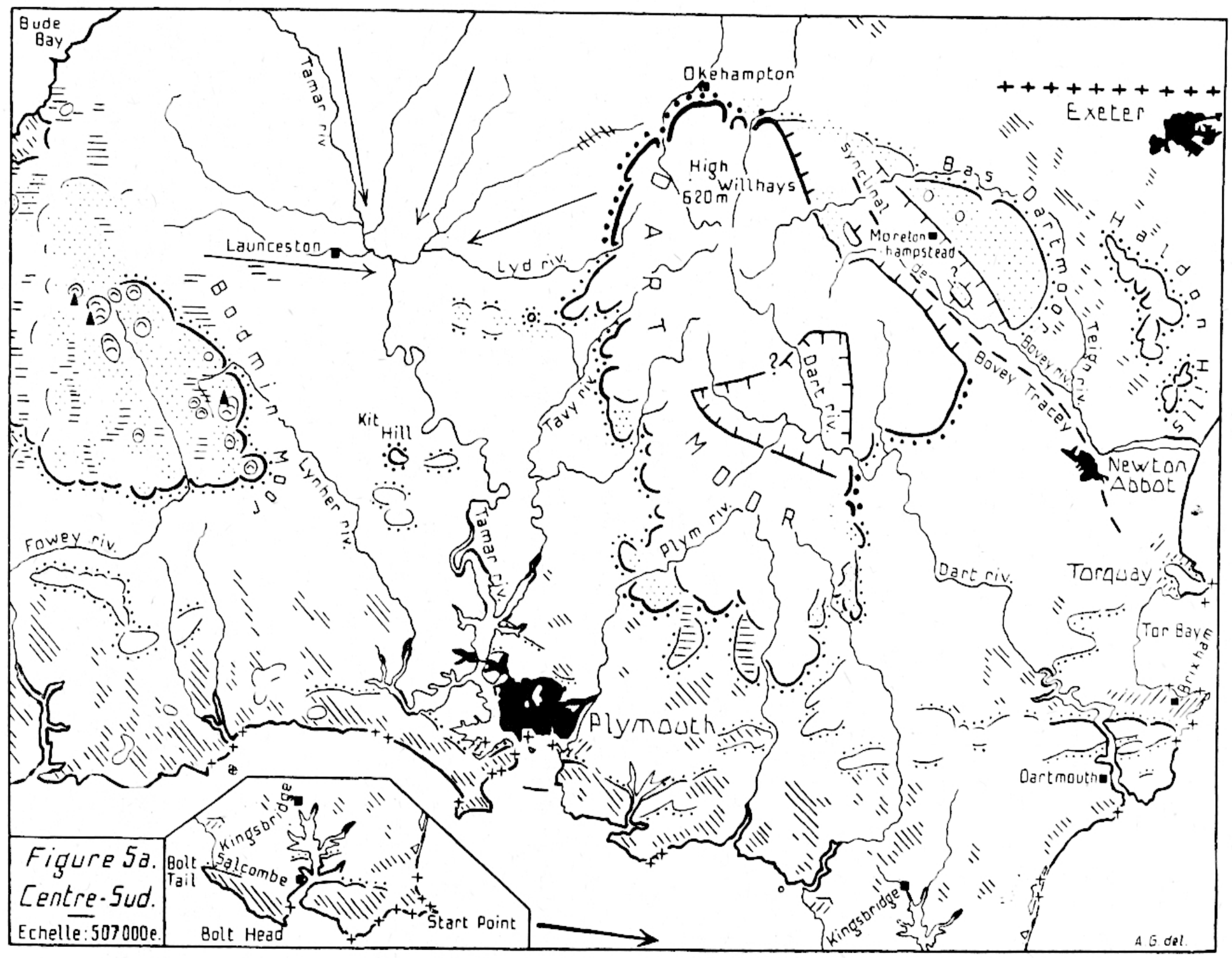




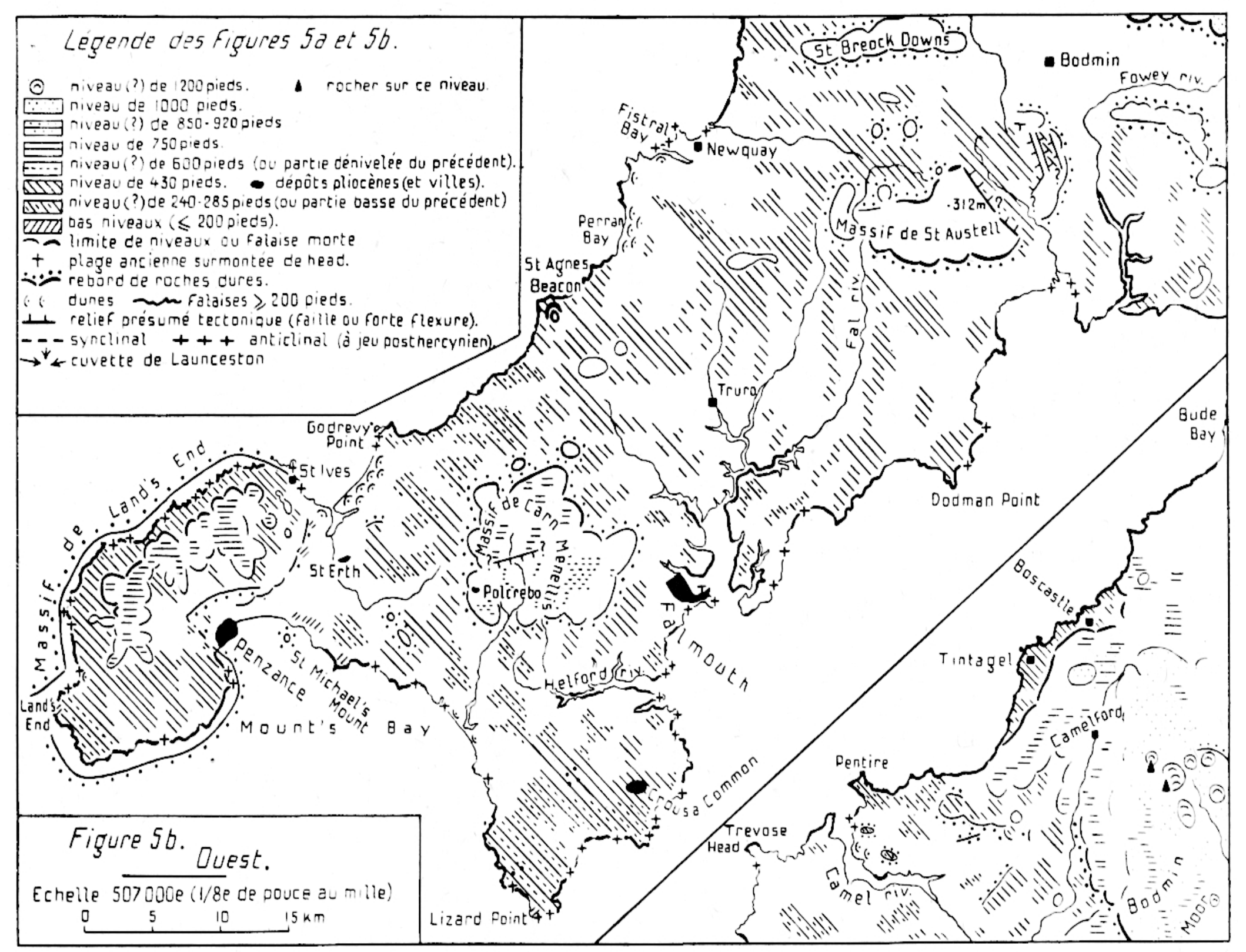


à ces endroits, deux dépressions NW-SE, parallèles au synclinal de Bovey Tracey et à la vallée rectiligne de la haute Fowey dans le Bodmin Moor, dont le fond est à $135-150 \mathrm{~m}$., et que limitent des croupes de $200 \mathrm{~m}$. ou plus. Ces larges sillons me paraissent avoir

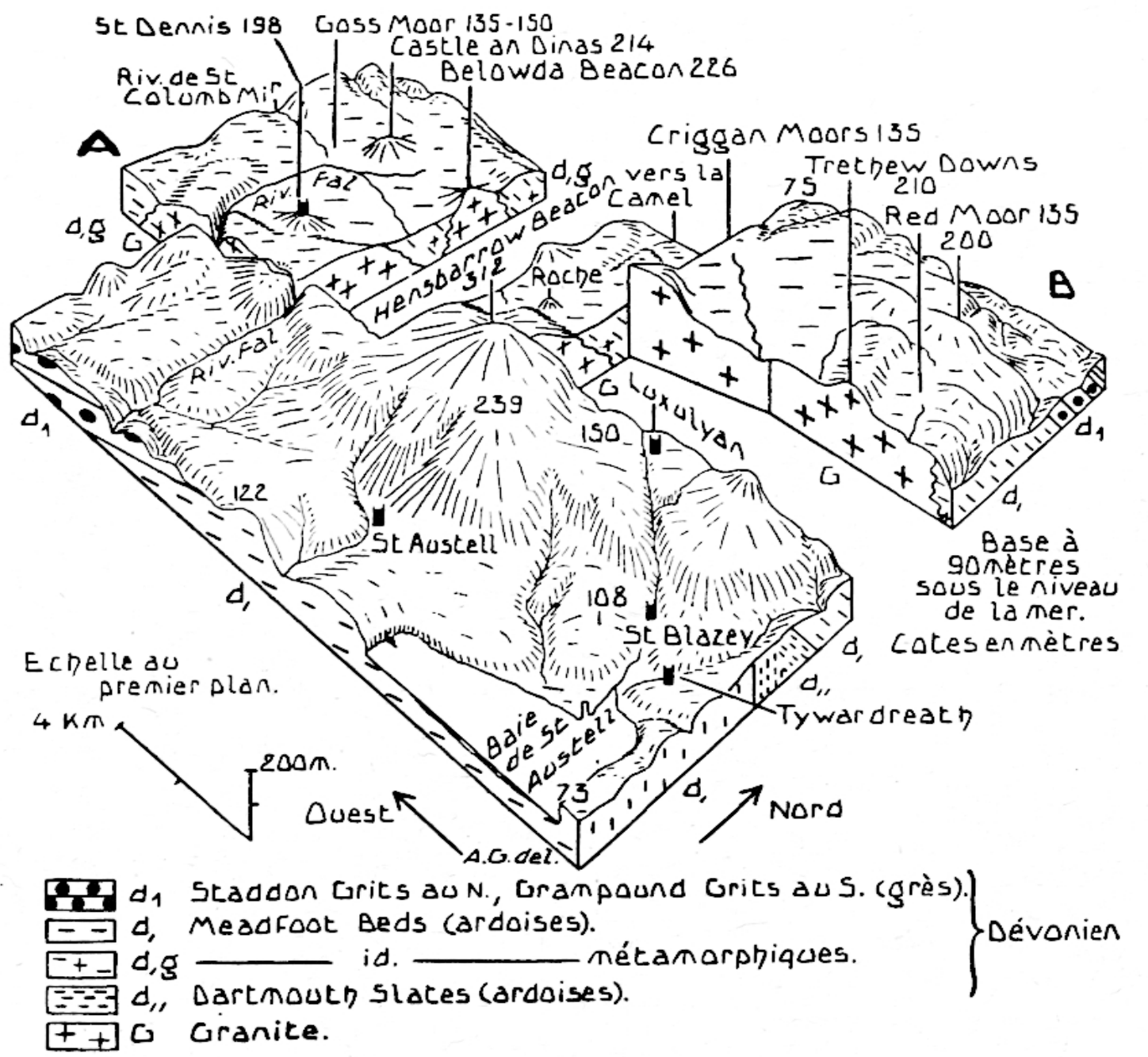

Fig. 6. - Bloc diagramme disséqué du massif de St Austell. Fragment $A$ tiré à $l^{\prime} W$ et $B$ au $N$, tous deux de $4 \mathrm{~km}$. Modifications récentes de l'écoulement des moors en $A$ et $B$; petites cheminées granitiques de St Dennis, Castle an Dinas, Belowda, Roche; dislocations vraisemblable; en $B$.

été longtemps drainés vers le Canal de Bristol par la Camel, dont aucune hauteur ne les sépare; l'écoulement vers la Manche par la gorge forestière de Luxulyan est sans doute récent. Mais les changements de drainage n'expliquent pas la topographie. Puisque le granite dans son ensemble forme un bombement comme aux massifs voisins, il est difficile de penser à une érosion différentielle 
aux dépens des roches cristallines sous un ancien climat tropical, idée émise pour d'autres pays d'Europe; la possibilité d'un jeu de failles autour de ces dépressions vient donc à l'esprit, renforcée par les correspondances d'alignements indiquées.

Le Dartmoor, bien plus grand que les autres bombements granitiques malgré ce qu'en a détaché le synclinal de Bovey Tracey, culmine à son extrémité $\mathrm{N}$, à $620 \mathrm{~m}$. à High Willhays. Il donne là, au-dessus d'Okehampton (fig. 5 a et 4 , petit bloc), les seuls reliefs montagneux de tout le massif Devon-Cornwall, comparables, avec une dénivellation de $480 \mathrm{~m}$., à ceux du Massif Central français. Ailleurs, les tourbières de pente ${ }^{16}$, la solitude, les empilements de blocs de granite à «dents de cheval » et à diaclases subhorizontales sur les tors et les carns viennent seuls suppléer, comme dans les Monts d'Arrée en Bretagne, aux fortes dénivellations pour évoquer la montagne. On pense donc à un basculement général N-S 17. Mais en outre il existe, au cœur du granite, une très curieuse dépression (fig. 5 a, et 4 , grand bloc) où la Dart s'attarde avant de dévaler dans les gorges boisées qui la mènent hors du cristallin. Cette haute cuvette tourbeuse, ressemblant au Yeun Elez (Marais de St Michel) de l'Arrée et au non moins funèbre Palais du Roi de la Haute Margeride, s'expliquerait assez bien par la tectonique. Ce n'est là qu'une hypothèse : le Professeur Stamp, à qui je l'ai soumise et qui connaît personnellement la région, y est peu favorable; il penserait plutôt à l'évidement d'une grande poche de kaolin. On vient toutefois de voir que, dans le massif de St Austell, plus kaolinisé, le relief ne s'explique pas par le degré d'altération du granite. La cuvette du Dartmoor semble appeler des recherches complémentaires. Si la présomption de failles venait à se vérifier, des dépôts superficiels pourraient se rencontrer dans la dépression, conservés sous la tourbe par affaissement.

\section{III. - Problèmes des surfaces étag̣ées.}

La littérature britannique concernant les surfaces étagées de ce massif est considérable, et une quasi-unanimité est faite à leur sujet : jusqu'à 1000 pieds $(300 \mathrm{~m}$.) environ, le massif est tronqué

16 On est en train de boiser l'Est du Bodmin Moor en conifères. Un Wald médio-européen vêtira-t-il un jour ces chauves mamelons ?

17 A l'W du Dartmoor, il en fut peut-être de même, si l'on en juge par le singulier report de la ligne de partage des eaux tout près de la côte $N$. Il y aurait en outre, me semble-t-il, une cuvette tectonique à Launceston (fig. 5 a). 
par des aplanissements non dérangés; la plupart des auteurs considèrent depuis très longtemps que ce sont sans doute des plates-formes d'abrasion marine; ils l'affirment tous pour la principale, qui s'étend à $300-430 \mathrm{p}$. (90-130 m.) environ. Bon nombre d'auteurs britanniques semblent d'ailleurs répugner un peu à l'idée de pénéplaine subaćrienne. Cette tendance doctrinale ne pouvait que rendre plus intéressant un nouvel examen.

Tout homme de bonne foi doit admettre deux choses avec nos confières: d'une part, l'étagement de surfaces non dérangiées est manifeste, et c'est une différence avec la Bretagne où, au-dessus de 100 no., la morphologie n'offre pas, du moins aux yeux de tous, une telle régularite; d'autre part, le niveau de $300-430$ p. est certainement une plate-forme d'abrasion marine. Mais l'érosion fluviale a laissé des traces non moins certaines de son travail en rapport avec cet ancien niveau de base, et aussi avec d'autres.

C'est sur le bord W, NW et $S$ du massif de Land's End, et près de 'Tintagel en Cornouaille du Nord, gue la plate-forme de 300430 p. est la plus belle. Elle s'y termine au pied d'une falaise morte splendide (fig. $5 \mathrm{~b}, 7,8,10 \mathrm{~F}$ ), et à Land's Fnd elle a fixé de nombreux villages au pied des hauteurs plus qu’à demi désertes qui la dominent. A St Agnes, elle a respecté une forte colline, singulièrement constituce de schistes alors qu'une petite intrusion granitique voisine a été totalement nivelée par exception (fig. $3 \mathrm{~F}$ ). Les formes seules indiquent une origine marine; mais les dépôts ne font pas défaut. Ie plus probant n'est pas, à notre avis, celui de St Erth, fossilifère marin du Pliocène inférieur, mais situé en contre-bas de la plate-forme ${ }^{1 s}$; c'est celui qui entoure le Beacon (colline) de St Agnes. Il est formé d'un sable rouge analogue à celui de St Erth et aux sables pliocènes marins de Bretagne ${ }^{19}$. Bien qu'azoüque, probablement parce que décalcilié, Reid, Milner et Macar le considèrent comme marin $2 \%$. L'échantillon que j'ai remis à A. Cailleux a conduit

1s Cl. Reid, The Pliocene Deposits of Britain. Mem. Geol. Surv., 1890, 326 p. (p. 59-66). - Ie dépôt, qui est diestien (plaisancien), se trouve dans la dépression entre Land's Fnd et Carn Menellis a 100-130 p. d'altitucie seulement; Reid $y$ voit néanmoins une preuve de lorigine marine de la plateforme, car, dit-il, il a dû se former à une profondeur de to fathoms environ étant donnés ses fossiles; $100 \mathrm{p}$. +40 fath. $=340 \mathrm{p}$. $(103 \mathrm{~m}$.$) . Il montre en$ tout cas que cette depression est prépliocène.

19 Sur ces sables, que l'on trouve jusyu'à $100 \mathrm{~m}$. d'altitude en Hautje Bretagne, ef. Guilcher, these, p. $556-563$ (état de la question en 1948 , et carte).

20 Reid, op. cit., p. 66-67. — Reid and Scrivenor, The Geology... near Newquay. EGS, 1906, 131 p. (p. 63-64). - Milner, The Nature and Origin of the Pliocene Deposits... JGS, IXXVIII, 1922, p. 348-377. - Macar, Sur Ia géomorphologie des Cornouailles... Bull. Soc. Giol. Belgiq., I.X, 1936-1937, p. $152-169$. 
ce géologue aux mèmes conclusions : les petits grains sont comparables à ceux de St Erth; les gros $(引 1$ mm) sont parfois éolisés, mais une usure marine a sùrement affecté le plus grand nombre. L'éolisation doit venir d'un remaniement quaternaire par le vent. On connait aussi de longue date des galets abondants et bien lités à 360 p. à Crousa Common, sur la plate-forme magnifiquement aplanie de Lizard (figs. 3 E), et d'autres beaucoup plus épars à Polcrebo sur le bord du massif de Carn Menellis:-1. A Polcrebo, l'altitude dépasse celle de la plate-forme (480 p. environ). I.es "Polerebo Gravels» sont considérés comme d'origine douteuse; mesurés par Cailleux, el d'après les termes de comparaison actuellement connus, ils montrent une dissymétrie fluviatile, peut-ĉtre un peu accrue par leur nature quartzeuse; leur indice d'émoussé les range aussi dans les galets lluviatiles. S'ils etaient marins, ce ne pourrait être qu'un dépòt en situation abritée; tel n'est pas le cas. Quant aux «Crousa Common Gravels», ils ont la même dissymétrie; ils sont deux fois plus émoussés, mais leur position hors de tout abri n'est pas non plus favorable à une origine marine: ils devraient être encore plus émoussés qu'ils ne sont, à moins que des causes de moindre usure encore iğnorées n'aient agi (là et à Polcrebo) :2. Jus(pu'ici, seule Miss Hendriks 2:3 arait vu dans Crousa Common un dépôt fluviatile. Rien ne s'oppose d'ailleurs à ce que la plate-forme qui les supporte soit marine, et le dépòt de St Agnes suffit a affirmer l'origine de la surface; sa disposition en aurécle autour du Beacon en fait une plage typique, qu'on peut dater du Pliocène par analogie avec St Erth.

En contre-bas de la plate-forme aboutissant a la falaise de 430 p., Balchin $\ddot{*}$ en voit une autre, vers l'estuaire de la Cannel entre 240 et 285 p. ( $72-86 \mathrm{~m}$.); il est exact qu'une telle surface bute a St Endellion contre un ressatut qui conduit au niveau de $430 \mathrm{p}$. (fig. 3 C, et 7 au contact du fragment $A$ et du bloc principal). I.e ressat correspond à des roches éruptives, ce qui ne l'empöche pas

21 Fleet and Hill, Geology of the lizard and Meneage. EGS, $2 *$ ed., 1946, 208 p. (p. 5-6 et 166-167). - I.e plateau de Lizard, admirablement conservé dans les serpentines, est plus disséqué dans le granite gneissique.

2 Dissymetries: Polcrebo: 0,589; Crousa (Common: 0.j90. Indices d'émoussé : $\mathrm{I}^{2}$ (longueur de $33 \mathrm{~mm}$ ) 0,064 (1.r ordre) et 0,079 (2* ordre); CG $(37 \mathrm{~mm}): 0.115$ et 0,146 . Plus fables indices d'enousse marin observes par Cailleux à $33 \mathrm{~mm}: 0,115$ et 0,135 . - - Sur lat signification de ces chiffres : Cailleux, Bull. Soce Géol. Fr., (5) XV, 1945, p. 375-404, et C. R. Sécule. Soce. Géol. Fr., 19+7, p. 250-252.

:3 E. M. Lind Hendriks, The Physiography of Southwest cornwall... Geol. Mag., I.X, 1923, p. 21-31.

24 WV. G. V. Balchin, The Erosion Surfaces of North Cornwall. Geogr. Journ, $\mathrm{XC}, 1937,2$, p. 52.63 . 
de pouvoir être un ancien rivage. Mais partout ailleurs, les plateaux descendant jusque vers 250 p. m'ont bien semblé n'être que la partie basse de la surface de $430 \mathrm{p}$. Un ressaut en un seul endroit n'est-il pas un critère d'individualisation insuffisant ? D'autre part, sur la côte SE, notamment vers Salcombe, la plate-formie de 430 p. passe, tout en restant plane, à des altitudes plus fortes. Sur la fig. 5 a j'ai indiqué le tout sous le même signe, mais il y aurait lieu de voir si une légère déformation récente n'est pas survenue de ce côté, ou si le niveau de $430 \mathrm{p}$. n'en remanie pas un autre qui serait un peu gauchi. En tout cas, rien ne correspond nettement au niveau de $80-100 \mathrm{~m}$. de Bretagne ${ }^{25}$, en dehors de la surface de 240-285 p., de Balchin.

Plus bas encore, Gullick ${ }^{26}$ semble avoir été le seul à s'intéresser en Cornwall à des replats côtiers à 180 p. (55 m.). Le bel aplanissement qui tronque les calcaires dévoniens à 200 p. $(60 \mathrm{~m}$. $)$ à Torquay et Brixham en Devon a été plus remarqué ${ }^{27}$. Tous semblent répondre à la «surface de Ste Anne»de Bretagne ${ }^{28}$. Une recherche soigneuse pourrait en révéler d'autres à cette altitude ou des altitudes différentes. De telles formes sont très nettes à Godrevy Point, Old Lizard Head (fig. 3 E, 5 b); à Plymouth, le nivellement des calcaires dévoniens de Hooe à 100 p. $(30 \mathrm{~m}$.) appelle aussi le rapprochement avec la Bretagne, comme le replat du golf de Thurlestone près de Kingsbridge (fig. 5 a).

Plus haut, les Anglais ont poussé davantage l'étude des niveaux. Gullick (op. cit., apud Steers) en voit sur le massif de Land's End à 750 p.; de près, la topographie paraît montueuse, mais d'un point éloigné les collines s'alignent en effet dans un même plan. J'ai trouvé ce niveau bien plus net vers Altarnum (NE du Bodmin Moor). Gullick le voit aussi dans le massif de Carn Menellis. L'examen de ce dernier mène à se demander s'il ne porte pas la trace de deux surfaces vers $225-230 \mathrm{~m}$. $(750$ p. $)$ et $180 \mathrm{~m}$. $(600$ p. $)$. On pourrait chercher si l'étagement ne répond pas à une dislocation, le niveau de 600 p. n'étant pas bien courant en Cornwall. Balchin (op. cit.) retrouve celui de 750 p. vers Boscastle, Camelford et plus au $S$, où il m'a paru très réel (fig. 5 b et 7 ).

25 Guilcher, thèse, p. 125-133, 144-155, 566-569.

se Gullick, A Physiographic Survey of West Cornwall. Trans. Roy-Geol. Soc. Cornwall, XVI, 1936, p. 80 (cf. Steers, p. 257).

27 Le plateau de Brixham ne peut guère être la surface prépermienne comme le suggère Ussher (Geology... around Torquay. EGS, 20 éd., p. 106-118) à cause des poches de Permien incluses dans le calcaire : il serait sûrement très disloqué par failles. Voir aussi les opinions de Jukes-Browne, op. cit. (1907) et Macar, op. cit., p. 165-169.

28 Guilcher, thèse, mêmes pages. 
Mais la plus nette de ces surfaces est celle de 900-1000 p. (270300 m.) ${ }^{29}$. Elle égratigne le $\mathrm{S}$ du Dartmoor, nivelle le Bas Dartmoor à part quelques buttes résiduelles (fig. 1), et surtout la plus grande partie du Bodmin Moor 30 (fig. 5 a, 7, $3 \mathrm{C}$ ), s'étendant indifféremment, dans le $\mathbf{N}$ de ce massif, au granite et aux roches sédimentaires et volcaniques. Le relief du Bodmin Moor m'a semblé se présenter

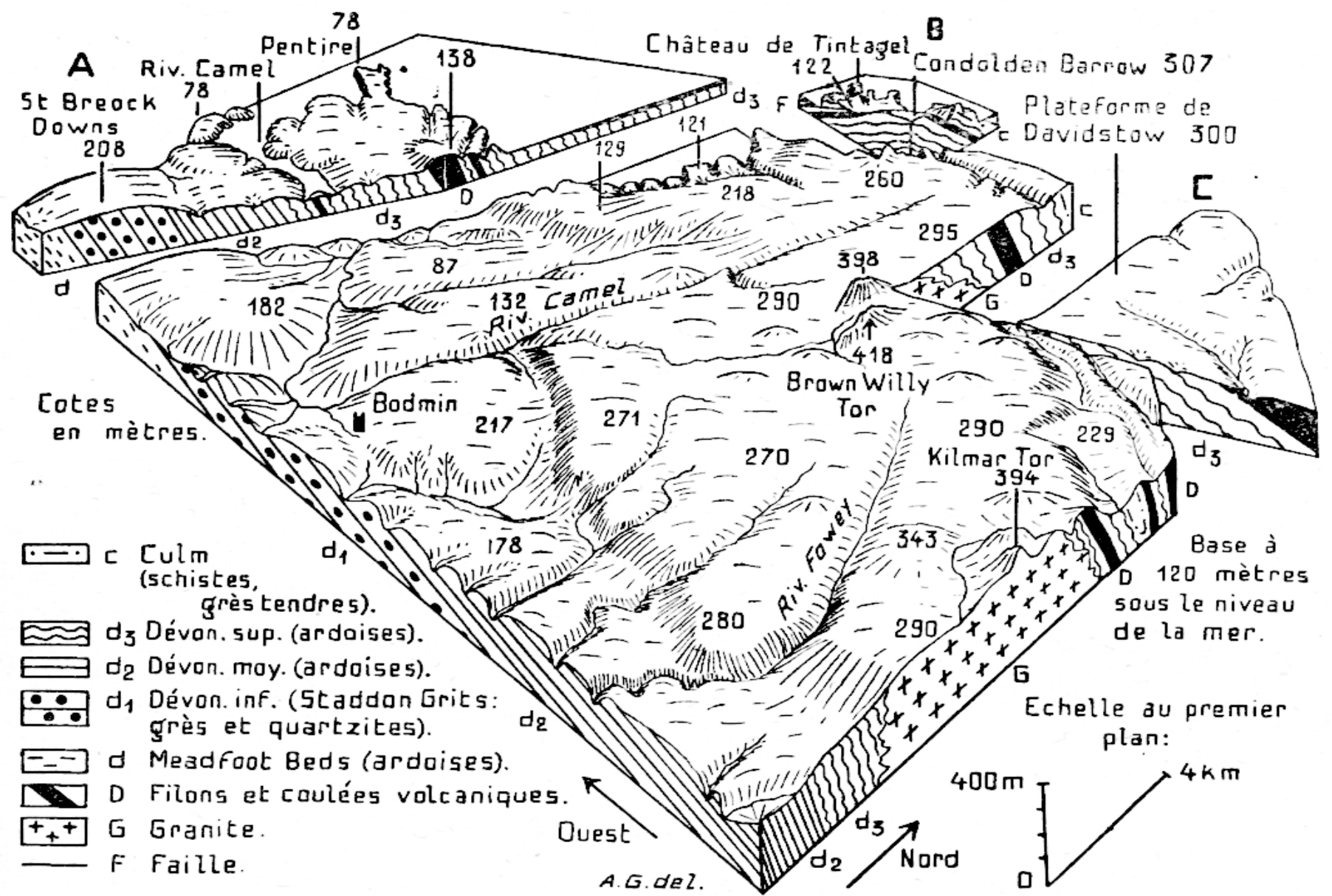

Fig. 7. - Bloc diagramme disséqué du Bodmin Moor et des niveaux étagés du $N$ de la Cornouaille. Partie A tirée à l'W, B au $N, C$ à l'ENE, toutes de 4 km. Etagement défilé par la perspective à Tintagel, mais visible plus au S. Bord du granite sans influence sur le relief au $N$ et $N W$ du Brown Willy Tor, mais très marque ailleurs. Deux zones de reliefs résiduels sur le moor. Rôle des Staddon Grits sensible à l'V, insensible vers Bodmin.

29 Balchin en dissocie, sans être très affirmatif, une surface de 850- $920 \mathrm{p}$. au $N$ de Camelford. surmontée par la butte de Condolden Barrow témoin de la surface de 1000. Il y a là en effet un étagement; mais le niveau de 850-920 est bien réduit et bien isolé.

30) Décrite là par Barrow. The High Level Platforms of Bodmin Moor... JGS, LXIV, 1908, p. 384-400. 


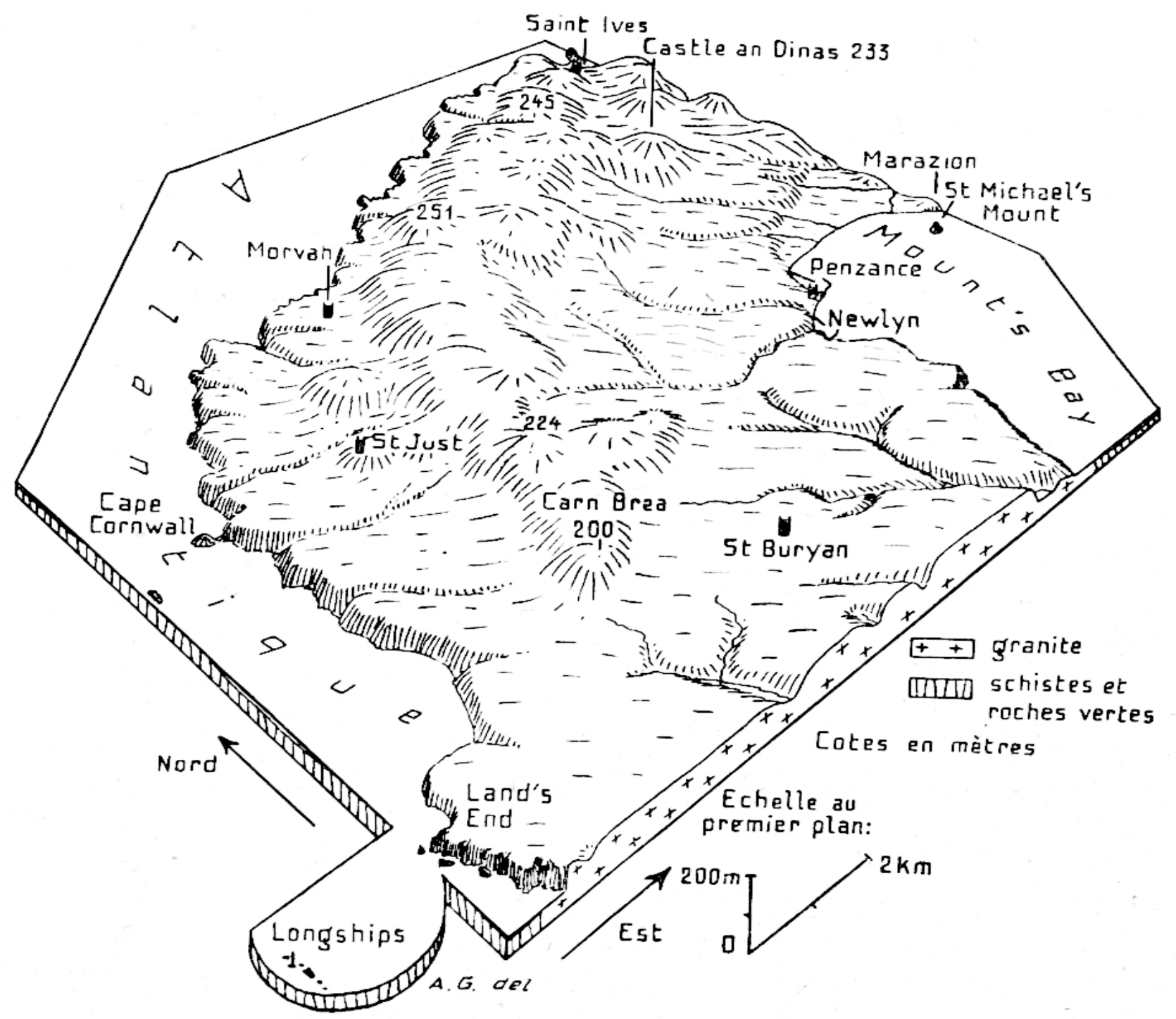

Fig. 8. - Bloc diagramme du massif de Land's End. Falaise morte à 439 pieds à $l^{\circ} W$, moors au-dessus; à l'E, niveau de 430 p. pénétrant dans les hav. teurs le long des vallées. Falaise morte monastirienne a NE de Penzance.

ainsi : la surface de 900-1000 p. a respecté, dans la région du Brown Willy Tor et dans celle du Kilmar Tor, une topographie de buttes très molles qui paraissent résulter de la dissection d'une surface de $1200 \mathrm{p}$. environ; au-dessus se dressent quelques rares rochers aux formes plus hardies, qui atteignent ou dépassent 1300 p. ${ }^{31}$. De même, les hauteurs de l'Exmoor disparaissent vers l'W devant une surface de 850-1000 p. portant quelques fortes

31 Macar avait déjà eu une impression analogue. 
buttes (Great Hangman, Holdstone Down, Trentishoe Iown) qui ne peuvent être que des reliefs résiduels de l'Exmoor.

I.e lecteur ne manquera pas de comparer ces surfaces avec celles reconnues en divers pays par $H$. Baulig. L'àge n'a jamais été fixé en Cornouaille; on peut seulement dire que la surface de 1000 p., qui ne paraît pas affectée par le synclinal de Bovey 'Tracey ${ }^{32}$, doit être post-oligocène. Mais un autre point a attiré mon attention. En plusieurs endroits, les niveaux sont prolongés à l'intérieur des terres plus hautes par de vieux fonds de vallées façonnés en fonction de l'aplanissement où ils aboutissent, et un peu plus élevés que lui. Ainsi, au $\mathbf{N}$ de Newbridge (W de Penzance) et au $S$ de $S t$ Ives entre Towednack et Halse Town, en fonction du niveau de 430 p.; ou encore la tourbière large et plate du Goss Moor, vers 450 p. entre le massif de St Austell et ses apophyses de Castel an Dinas et Belowda (fig. (6, partie A), qui dut être modelée par la tête du cours d'eau de St Columb Minor avant d'être captée par la Fal; sur le bord $W$ du Bodmin Moor, vers Blisland, plusieurs replats représentent aussi le niveau de 430 p.; à l'intérieur du mème massif, le niveau de 1000 p. est entaillé de vallons du cycle de $750^{33}$, et celui de 1200 p. par des vallons du cycle de 1000. Quelle que soit l'origine des hautes surfaces, qu'on soit ou non eustaticien, la classique prolongation de la plate-forme vers l'amont par des replats ou fonds de vallées est bien réalisée. Resterait à préciser et dater tout cela, ce qui serait plus difficile que de citer des formes notées au passage. Ce travail a déjả été tenté ${ }^{34}$, mais, avant de le reprendre, un recensement complet dans le massif ancien serait nécessaire. Resterait aussi à étudier de près le tracé de la côte, l'origine de la dépression sublittorale ennoyée de Falmouth ${ }^{35}$, et à continuer l'étude du réseau hydrographique déjà bien entamée. Là encore il doit y avoir des analogies avec la Bretagne.

$3:$ Il se pourrait qu'au $S$ dokehampton le bord du Dartmoor se soit soulevé depuis l'époque du niveau de 1000 p.; le plateau à $1150-1200$ p. (360 m.: figs. 4, petit bloc) qui porte les batiments du camp, à l'altitude asse\% inhahituelle, suggère cette idée.

:: Barrow a vu a moins celui de Trebartha, dans le NE.

34 Green, The terraces of Southernmost England.

3.) Osman (The Granites of the Scilly Isles... JGS, IXXXXIV, 1928, p. 258292 ; cf. p. 289) a parlé de subsidence pour Falmouth et Plymouth, sa!̣s donner de preuves. Sur Plymouth, cf. supra, note 13. 
IV. - Iroblèmes du littoral et du guaternaire rérent.

C'est par les côtes que le relief de ce massif ressemble le plus au relief breton. L'âpreté et la noblesse des paysages sont pourtant encore plus marquées. L'altitude des falaises est plus forte : elle n'est que de 100 à $110 \mathrm{~m}$. au maximum en Bretagne (Presqu'île de Crozon, Goello); elle peut atteindre et dépasser $250 \mathrm{~m}$. dans le $\mathrm{N}$ du Devon (fig. 10, A), où les hog's backs (dos de porcs) au profil arrondi dans les Hangman Grits sont, dit-on, plus imposants que n'importe quelle côte salloise. Le déchiquètement des raides falaises de serpentine dans l'W de Lizard, tronquant un plateau régulier (Demangeon, pl. XXXVII B), évoque Belle Ile. Les dunes montueuses des baies de Perran et St Ives rappellent les amas de sable du Léon ou de Quiberon, et ont sans doute requis comme eux une alimentation plus grande qu'aujourd'hui pour leur formation. Les petits étangs de barrage analogues à ceux de Bretagne méridionale ne sont pas inconnus (fig. 5 a et b). Mais très rares sont les secteurs bas en dehors de quelques baies comme Mount's Bay. Il n'y a de correspondance qu'avec le $N$ et l'W de la Bretagne, non avec le $\mathrm{S}$. Conséquence normale de l'altitude plus forte en tous ses points du massif britannique.

L'évolution récente des deux littoraux offre une quasi-identité. L'érosion marine actuelle est le plus souvent impuissante en Bretagne. Elle l'est aussi en Cornwall-Devon, comme l'avait souligné Demangeon. Le massif de Laand's End, exposé en plein Ouest, recèle dans les pans de son granite ruiniforme (castellated : fig. $10 \mathrm{D}$ ) de nombreuses plages suspendues dont les galets colossaux ont jusqu'à 1 mètre (fig. 5 et $9 \mathrm{~A}$ et $\mathrm{B}$ ). Là où je les ai examinées, les plages anciennes, très bien inventoriées 36 , m'ont toujours paru correspondre à ce qu'on appelle en France le Normannien ou Monastirien : elles sont à quelques mètres au-dessus des hautes mers actuelles, reposant sur une plate-forme d'abrasion en voie d'exhumation (fig. $9 \mathrm{D}$ ), et surmontées par une formation de solifluxion nommée head ${ }^{37}$ qui s'écoula sous un climat de toundra.

30 Bibliographie très copieuse. Tableaux d'ensemble dans Prestwich. The Raised Beaches...JGS, XLVIII, 1892, p. 263-343, et Green. The Age of the Raised Beaches... PGA, LIV, 1943, p. 129-140. — Ces plages recèlent très souvent des silex comme celles d'Armorique. Quelques auteurs se sont beaucoup tracassés à ce sujet; les silex doivent être venus tout simplement de fonds voisins, ou par glaces flottantes comme certains blocs exotiques.

37 Le mot head (= sommet des couches) a été employé d'abord en Cornwall par le génial De La Beche, qui dès 1839 décrivait, dans son Report on the Geology of Cornuall, Devon and West Somerset, bien des choses que l'on a mis cent ans de plus à voir en Bretagne. 


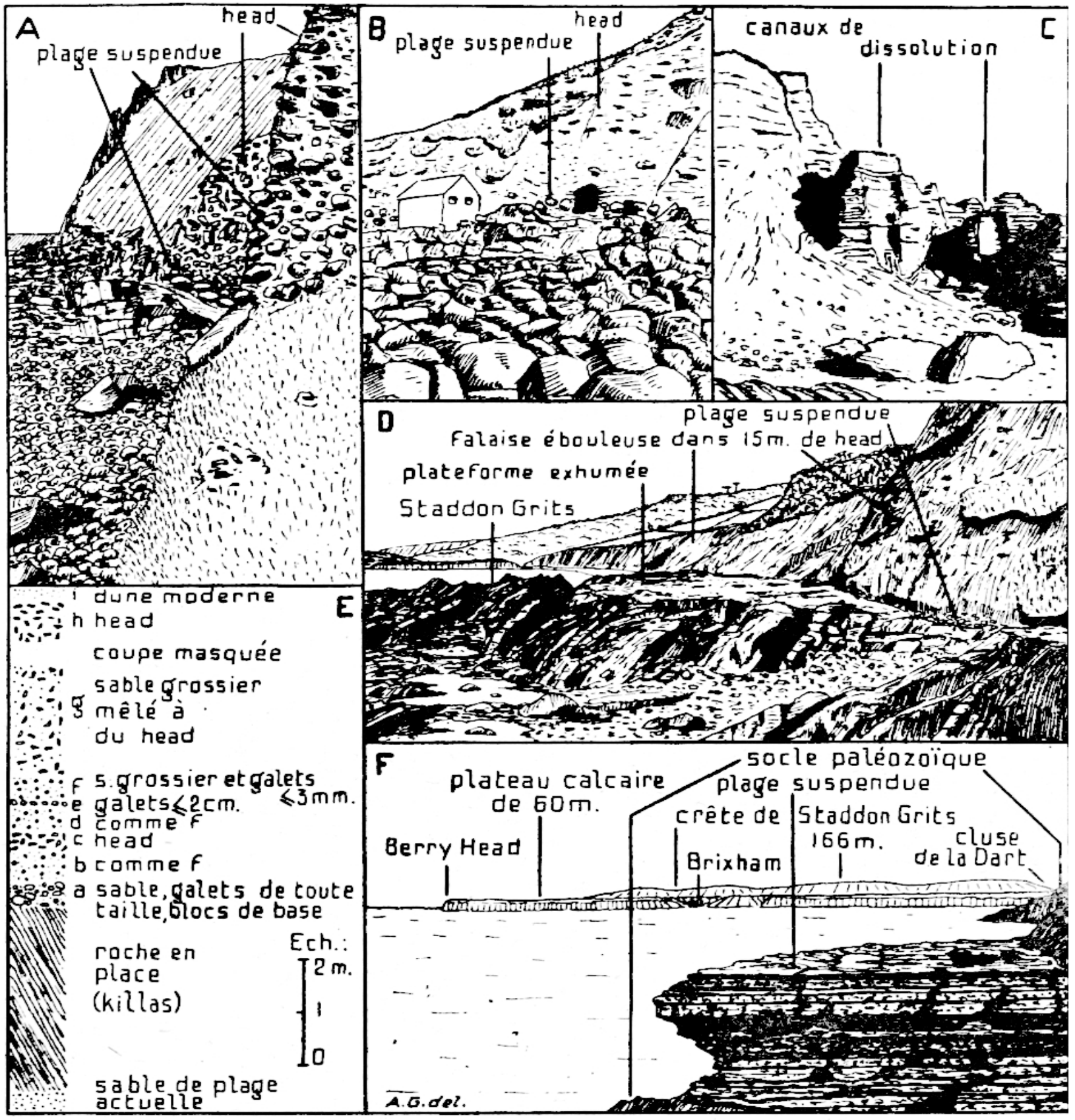

Fig. 9. - Formes et dépôts littoraux anciens (croquis d'après nature). A : Porth Nanven (S du Cap Cornwall). La falaise abrupte au fond a $90 \mathrm{~m}$. de. haut. - $B$ : Priest's Cove, sous le Cap Cornwall. - C : Fistral Bay, partie $N$ Cylindres de dissolution dans un sable ancien marin et dunaire. - D : Picklecombe (rade de Plymouth). - E : Fistral Bay, coupe dans la partie S. - I* : Hope's Nose (Torquay). Vue ver's le S. Niveau de 200 p. au fond. 
Elles sont donc antérieures à une phase glaciaire, el par suite à la régression préflandrienne qui correspondit, grosso modo, à la dernière glaciation. Même le «Haut Monastirien» (12-20 m.), connu en Bretagne méridionale en plusieurs points, est représenté à Plymouth et à Newlyn près de Penzance. L'abondance de ces plages, qui est considérable tant en Armorique (Bretagne et Normandie) qu'en Devon-Cornwall, prouve l'extrême faiblesse du sapement marin dans les deux massifs depuis la transgression flandrienne, les lignes des rivages monastirien et actuel étant pratiquement en coïncidence. Ces plages ne sont pas toujours décalcifićes comme les nôtres : ainsi celle de Hope's Nose (figg. 9 F) est extrêmement coquillière, reposant il est vrai sur du calcaire dévonien. I'autre part, sur les côtes $\mathbf{N}$ de Cornwall et de Devon, l'intercalation d'une dune ancienne entre la plage et le head est très fréquente : ainsi à Fistral Bay, où un sable au moins en grande partie dunaire est parcouru de «pipes» verticales cylindriques de dissolution (fig. $9 \mathrm{C}$ ). Plus importantes sont les conclusions récemment tirées par Arkell de la coupe de Trebetherick sur l'estuaire de la Camel ${ }^{38}$. Il y aurait là d'après lui deux plages et deux coulées de head successives d’àges différents, et la plage inférieure serait de l'avant-dernier interglaciaire. Ces conclusions n'ont pas emporté l'adhésion de tous ${ }^{39}$; je n'ai pas vu les lieux, mais la coupe que j'ai relevée dans le $\mathrm{S}$ de Fistral Bay et qui offre des alternances de ce genre (fig. $9 \mathrm{E}$ ) me paraît pouvoir s'expliquer comme certaines successions des côtes bretonnes : coulées de head en partie contemporaines de l'édification des cordons monastiriens ${ }^{40}$. Il y a pourtant là un problème que je me propose de revoir de près dans le $\mathbf{N}$ de ce massif et le $\mathrm{S}$ du massif gallois. Mais même si la plage inférieure était par endroits prérissienne, le façonnement de la côte n'en serait pas plus récent, au contraire.

Le profil des falaises aussi parle le plus souvent de stabilité. Les fig. $10 \mathrm{~A}, 13, \mathrm{D}$ montrent un simple lessivage actuel des parties basses. La fig. $10 \mathrm{C}$ fait voir le contraste entre la falaise de roches dures, typique du massif ancien, et les glissements qui emportent tout le relief continental dans le New Red Sandstone. Il est certain que, dans cette dernière roche, le recul est aussi grand que dans la craie où, à l'E de Seaton, un éboulement de 8 millions de tonnes a

:s W. J. Arkell, The PJeistocene Rocks at Trebetherick Point... PGA, I,IV, 1943, p. 141-170.

: Notamment celles de Bull et Green (Arkell, discussion in fine). Pour Green, toutes ces plages équivalent à notre Monastirien. Cf. Green, The Agé of the Raised Beaches...

40 Guilcher, thèse, p. 225-226 et 582-583. 


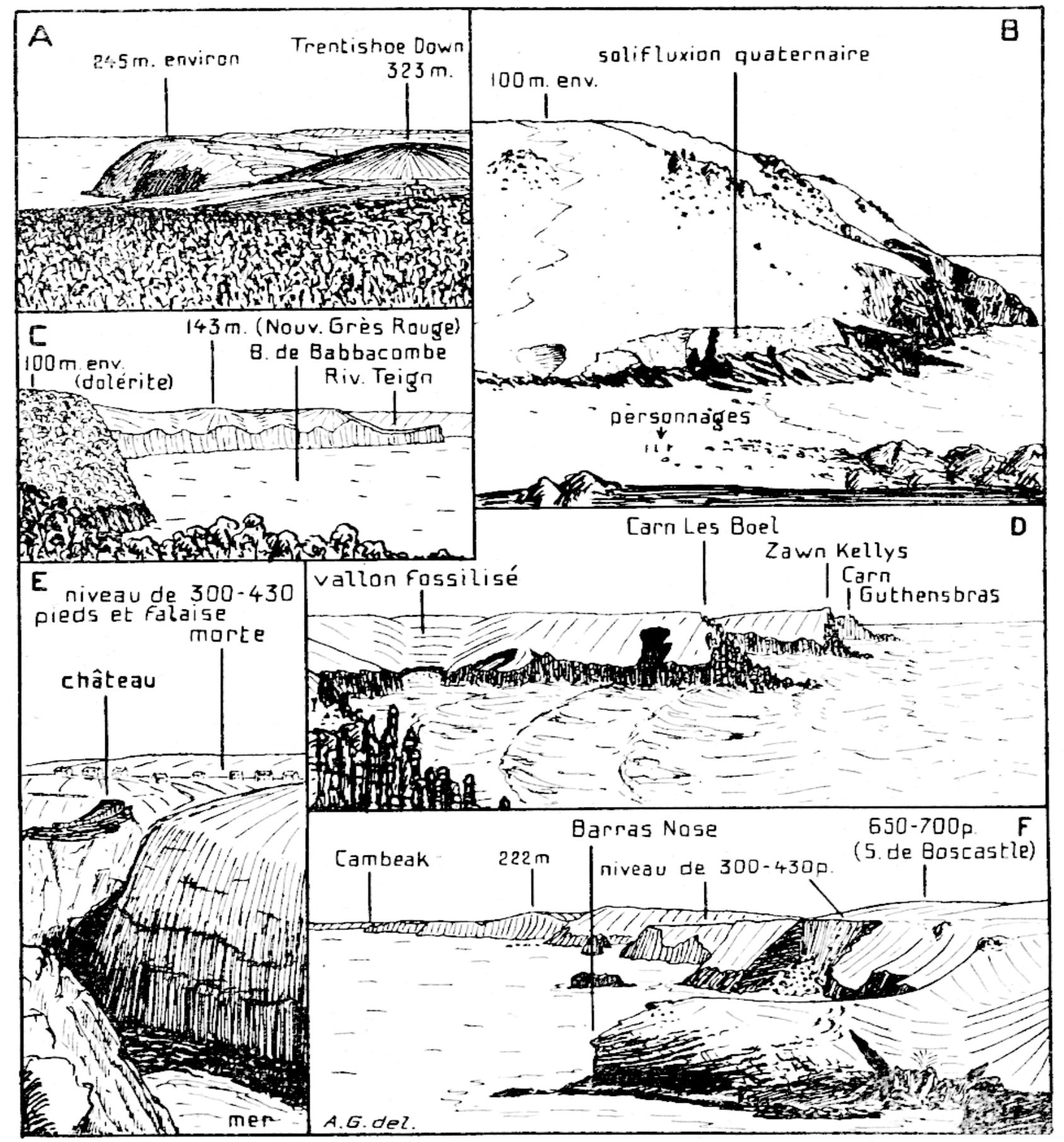

Fig. 10. - Falaises (croquis d'après nature). A : Hog's backs du $N$ du Devon (Hangman Grits). - B : Crique de Sewer (côte de Bolt, S Devon). Schistes. - C : De Hope's Nose (Torquay) vers le $N$. - I) : Au S de Land's End. Hauteur des pointes : 65 à $70 \mathrm{~m}$. Granite. - F : 'Tintagel. Roches volcaniques. - F : de la prestu'île de Tintagel vers le $\mathrm{N}$. Roches voleaniques, puis schistes au fond. 
ouvert en 1839 une gigantesque plaie qui se voit toujours ${ }^{41}$. Même contraste sur les deux rives de la Manche.

Pourtant, quelques secteurs du massif ancien ont dû souffrir un peu plus que l'ensemble. Sur la côte $N W$ et $N$ de Cornwall, l'avivement de la falaise sur toute sa hauteur n'est pas rare (fig. $10 \mathrm{~F}$ ), et entre Trebetherick et la baie de Barnstaple il n'y a plus de plages suspendues. Flett et Hill insistent sur la jeunesse des falaises de serpentine au NW de Lizard. Mais il ne faudrait pas induire de la verticalité d'une falaise qu'elle recule forcément beaucoup. A Tintagel (fig. $10 \mathrm{E}$ ), le château dit du Roi Arthur surmonte un gouffre vertigineux pouvant être considéré à première vue comme en pleine évolution; mais le mur qui domine le précipice est de la fin du XIv siècle; or, quand on l'a fait, la falaise avait le tracé actuel, car le site aurait perdu sa valeur s'il y avait eu moyen de passer entre le mur et l'abrupt. Plusieurs pointes déchiquetées portent des enceintes de défense préhistoriques qui mènent au même raisonnement.

L'étude des vallons côtiers offre le même intérêt qu'en Bretagne pour l'évolution de la côte. Les vallons suspendus sont bien plus fréquents ici parce que le rivage est plus souvent élevé. Mais ils n'ont été analysés que sporadiquement, sauf ceux de la côte $\mathbf{N}$ qu'Arber a inclus dans sa grande étude ${ }^{42}$. Les types que j'ai essayé de définir en Bretagne ${ }^{43}$ m'ont bien semblé exister ici aussi. Le «vallon suspendu eustatique», qui était raccordé au niveau monastirien et est resté suspendu par suite de l'abaissement postmonastirien du niveau marin, existe à Tremearne par exemple (fig. $11 \mathrm{D}$ ), une plage monastirienne se trouvant à l'embouchure. Celui de Trebarwith au $S$ de Tintagel en est un autre, avec cran de raccordement dans le plafond monastirien. Les vallons suspendus par recul de la côte, du type du Pays de Caux, sont très nombreux dans le Secondaire; celui de la figure $11 \mathrm{~A}$, près de Torquay, est curieusement sectionné par la tête. Mais on en voit aussi dans le massif ancien : à Bolt Tail, la mer ronge une tête de vallée comme à Torquay; près de là, à Hope, elle a capté un ruisseau dans son cours inférieur (fig. $11 \mathrm{~B}$ ); juste au $\mathrm{S}$ de Land's

41 Cf. Demangeon, pl. XL A. - Le recul est pourtant inégal. Un rocher de New Red à Thurlestone (côte de Bolt) avait dès le $1 x^{\mathrm{e}} \mathrm{s}$. Sa forme actuelle (Arkell, p. 163); et il $y$ a une plage suspendue antérieure au head à l'entrée de l'Exe dans le New Red (Prestwich, p. 279).

4.2 Arber, The Coast Scenery of North Devon. Londres, 1911 (cf. Steers). Dewey, On the Origin of some River Gorges... JGS, LXXII, 1915-1916, p. 63-76. - Hendriks, op. cit. - Macar, op. cit.

43 Guilcher, thèse, p. 237-250 et 424-434. 


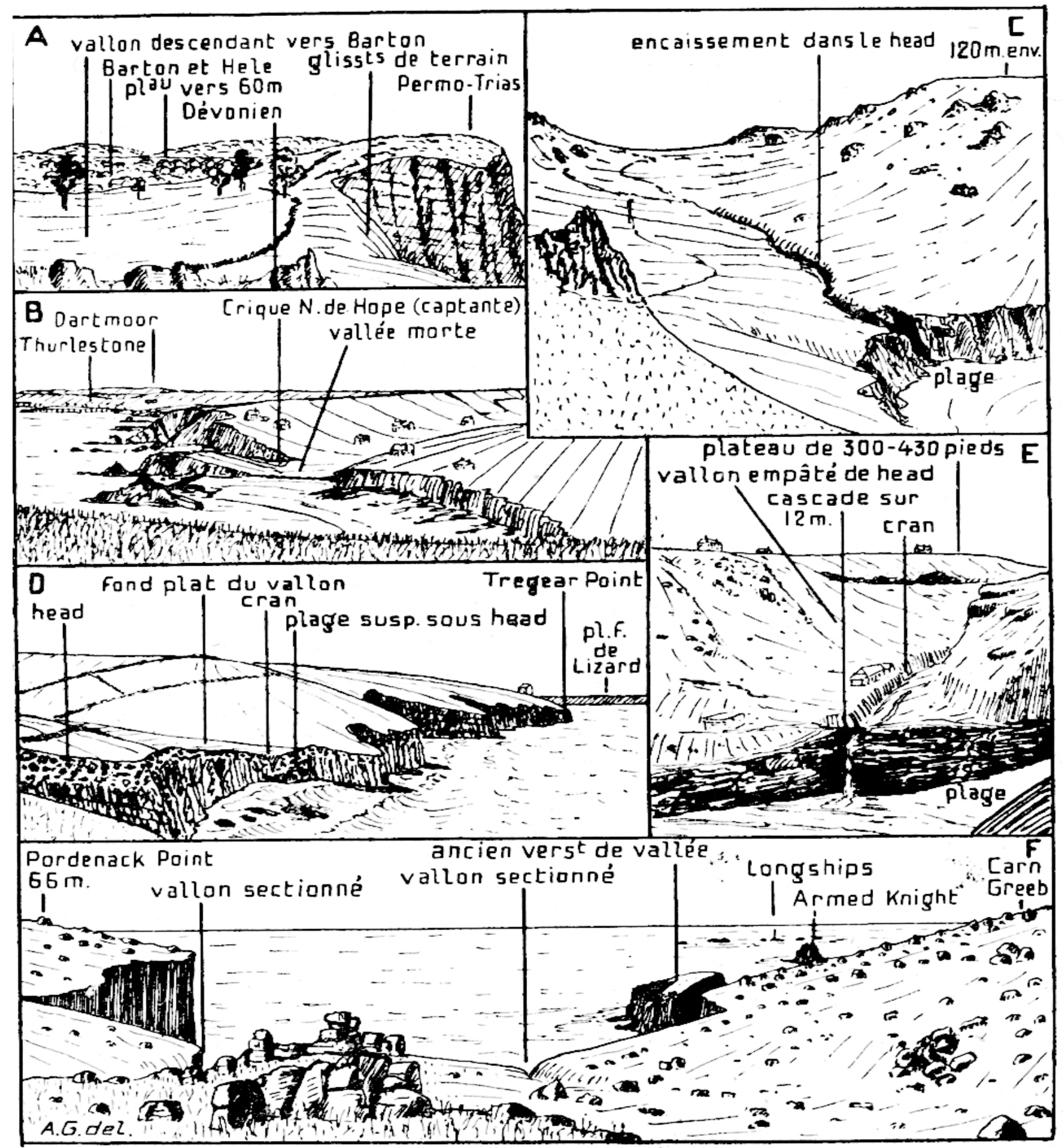

Fig. 11. - Vallons côtiers (croquis d'après nature). A : Oddicombe (Torquay). - B : Hope (près de Salcombe, Devon). - C : Sewer Valley (près de Salcombe). - D : Tremearne (entre I,izard et Penzance). - E : Tintagel. F : Face $S$ de Land's End. 
End, le cours inférieur commun de deux ruisseaux a été emporté, et du versant droit de la vallée détruite ne reste qu'un lambeau isolé (fig. $11 \mathrm{~F}$ ). Arber a cité des cas de ce second type dans le $\mathrm{N}$. Mais une partie de ces conquêtes marines peut bien dater du Monastirien. Tous les vallons ont été fossilisés par des coulées qui leur donnent un profil d'auge, un fond plat parsemé de blocs et souvent entaillé d'une gorge de raccordement récente (fig. 11, C, D, E, F; fig. $10 \mathrm{D}$ ); à Tremearne (fig. $11 \mathrm{D}$ ), la gorge ne retrouve pas l'ancien talweg, et il y a épigénie en miniature sur le head comme dans le Cap Sizun en Bretagne. Les exploitations stannifères ont souvent réalisé une exhumation artificielle, car l'étain s'était concentré à la base du head.

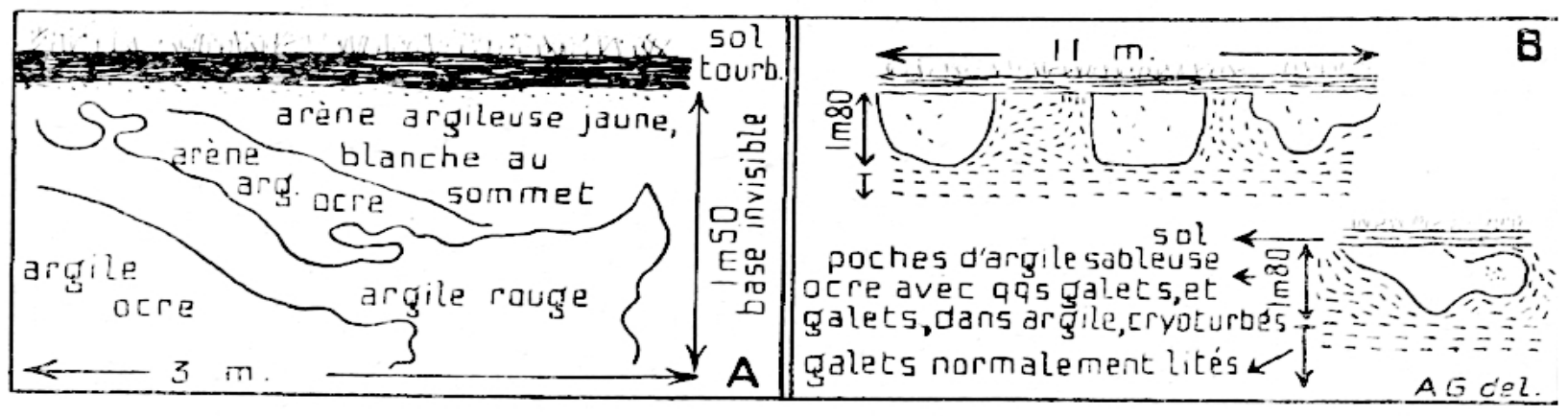

Fig. 12. - Cryoturbation quaternaire (croquis d'après nature). A : sol injecté et plissé près du Colvannik Tor (Bodmin Moor). Altitude: $250 \mathrm{~m}$. 13: Partie superficielle cryoturbée du dépôt de Crousa Common (Iizard).

La cryoturbation ${ }^{44}$ quaternaire, qui empâte l'intérieur comme la côte, est plus épaisse qu'en Bretagne; elle peut dépasser $15 \mathrm{~m}$. et même, paraît-il, parfois $30 \mathrm{~m}$. Mais ses formes sont généralement plutôt banales : essentiellement des coulées d'argile à blocs. J'ai pourtant noté sur le Bodmin Moor (fig. 12 A) un bel exemple de sol injecté et plissé, et, dans la partie supérieure du dépôt de Crousa Common (fig. 12 B), des sortes de poches qui, si elles étaient vues en section horizontale, pourraient se révéler être un sol polygonal. Jusqu'ici on n'a pas décrit de polygones fossiles en Cornwall à ma connaissance, alors qu'il y en a de splendides en Bretagne ${ }^{4 \pi}$.

4t Phénomènes dus au dégel superficiel estival, affectant les pays frojds sans glaciers et à sous-sol toujours gelé. Cf. A. Cailleux, Etudes de Cryopédologie. Paris, Tournier et Constans, 1948, 68 p.

45 Iiste, description et références dans Guilcher, thèse p. 441-442. Y ajouter les polygones de la plage du Curnic (IV de Beg ar Skez) en Guisseny (Nord Finistère), que j’ai trouvés avec $P$. R. Giot en août 1948 et qui sont les plus heaux de tous. 
Les épisodes de creusement sous le niveau actuel sont les mêmes qu'en Bretagne. La profondeur du lit rocheux des rias sous la vase, mesurée en de multiples endroits, est en général d'une trentaine de mètres près de l'embouchure ${ }^{46}$. Mais les rivières n'ont pas creusé sous le niveau actuel qu'entre le Monastirien et le Flandrien; avant le dépôt des plages suspendues, elles l'avaient déjà fait comme en Bretagne: les estuaires de Salcombe, de Plymouth et de la Camel sont certainement antérieurs aux plages suspendues, puisfu'ils en portent dans leurs entrées. Il en va d'ailleurs de nême pour la rade de Cork en Irlande du Sud ${ }^{47}$.

Que de retards et d'hésitations auraient été épargnés à ceux qui se sont occupés du Massif Armoricain s'ils avaient regardé plus tôt vers le pays frère! Que de questions gagneront encore à être vues des deux côtés de la Manche à la fois ! Mais s'il faut mettre une idée en valeur pour finir, ce sera la faiblesse de l'érosion marine aux dépens des saillants ${ }^{4}$ du massif de Devon-Cornwall depuis la dernicie grande transgression; et l'on dira avec De La Beche: «Quand nous voyons tant de roches dures ciselées d'anfractuosités et de criques, et apprenons, comme nous pouvions l'attendre en effet de leur aspect, que l'on n'a observé de mémoire d'homme aucun changement appréciable en elles, nous pouvons croire volonlier's que l'état actuel de cette côte n'a pas été réalisé pendant une durée qui soit à l'échelle humaine ${ }^{49}$.

Dans le $N$ du Devon, de multiples replats discontinus, coupant la pente de hautes collines, sont peut-être aussi une forme de cryoturbation, qui serait à rapprocher des « replats goletz» (cf. Rev. de Géogr. Alpine, XXI, 1933, p. 347-371). Ils sont étudiés, en relation avec des formes analogues des Monts d'Arce, dans un article sü ces derniers (Ann. de Bretagne, 1949, sous presse). 278.

(1" Codrington, On some Submerged Rock Valleys... JGS, LIV, 1898, p. 251-

47 Reid and Scrivenor, op. cit., p. 69-71. — Ussher, The Geology... around Kingshridge and Salcombe. EGS, 1904, p. 71. — Id and Flett, The Geology... a!ound Plymouth and Liskeard. EGS, 1907, p. 118 et 127. - Arkell, op. cit., p. 146. - Wright and Muff, The Pre-Glacial Raised-Beach of the South Coast of Ireland. Scient. Proceed. Roy. Dublin Soc., n. s., X, 1904, p. 250-324.

is Des saillant;, car la mer avance dans certaines baies formees de roches teudres : Mount's Bay (Reid and Flett, The Geology of the Land's End District. EGS, 1907, p. 83.), Tor Bay (Ussher, The Geology... around Torquay. EGS. 2 ed., 1933, p. 119). A Mount's Bay, où la mer progresse vers unc falaise morte monastirienne, l'analogie avec la Bretagne est totale.

4: L'autcur est confus de s'être cité lui-même si souvent dans cet article. Il l'a fait, d'une part pour ne pas revenir en détail sur des faits longuement exposés ailleurs en ce qui concerne la Bretagne, d'autre part pour ne pas laisser sans références le lecteur non spécialisé dans ces problèmes.

Note ajouté i l'inipression. - Un second voyage (juillet-août 1949) m'a permis d'examiner la plage suspendue de Trebetherick etudiée par Arkell (cf. supra), et celles du $N$. du Devon, du Bill of Portland, de Gower (Galles), de Jersey et (iucruesey. Toutes sont, à mon avis, monastiriennes (sauf celles à plus de $20 \mathrm{m.}$; je ne suis donc pas Arkell. Ie m'en expliquerai ailleurs plus tard. 